\title{
GALOIS MODULE STRUCTURE OF UNRAMIFIED COVERS
}

\author{
GEORGIOS PAPPAS*
}

\section{INTRODUCTION}

Let $G$ be a finite group. Suppose that $Y$ is a projective algebraic variety over $\mathbf{Z}$ (i.e an integral scheme which is projective and flat over $\operatorname{Spec}(\mathbf{Z})$ ) of relative dimension $d$. In this paper, we consider finite Galois covers $\pi: X \rightarrow Y$ with group $G$ which are everywhere unramified, i.e " $G$-torsors". Let $\mathcal{F}$ be a $G$-equivariant coherent sheaf on $X$. Consider the value of the right derived global section functor at $\mathcal{F}$. This is a complex $\mathbf{R} \Gamma(X, \mathcal{F})$ in the derived category of complexes of modules over the group ring $\mathbf{Z}[G]$ whose cohomology groups are the finitely generated $G$-modules $\mathrm{H}^{i}(X, \mathcal{F})$. The central question in the theory of "additive" Galois module structure is:

Existence of a normal integral basis: Is the complex $\mathbf{R} \Gamma(X, \mathcal{F})$ isomorphic to a bounded complex of finitely generated free $\mathbf{Z}[G]$-modules?

By an observation of Chinburg [C] (which essentially goes back to Noether), the complex $\mathbf{R} \Gamma(X, \mathcal{F})$ is always perfect, i.e is isomorphic to a bounded complex of finitely generated projective $\mathbf{Z}[G]$-modules. It follows that the obstruction to a positive answer to our question is given by the "stable projective Euler characteristic" of $\mathbf{R} \Gamma(X, \mathcal{F})$. By definition, this is an element $\bar{\chi}^{P}(\mathcal{F})$ in the class group $\mathrm{Cl}(\mathbf{Z}[G])=\mathrm{K}_{0}(\mathbf{Z}[G]) / \pm$ free classes $\}$ of finitely generated projective $\mathbf{Z}[G]$-modules.

Let $N / K$ be a Galois extension of number fields with group $G$ which is unramified at all finite places. Then we can take $X=\operatorname{Spec}\left(O_{N}\right), Y=\operatorname{Spec}\left(\mathcal{O}_{K}\right)$, with $\mathcal{O}_{N}, \mathcal{O}_{K}$ the corresponding rings of integers. Our question for $\mathcal{F}=\mathcal{O}_{X}$ amounts to asking if the ring of integers $\mathcal{O}_{N}$ is a stably free $\mathbf{Z}[G]$-module; this type of problem has a long history going back to Hilbert and Noether. By "Fröhlich's conjecture" (shown by M. Taylor; see [Ta], $[\mathrm{F}]$ ) the answer is positive if in addition either $G$ is abelian or $N / K$ is also unramified at the infinite places. Without these assumptions it is known that $\mathcal{O}_{N} \oplus \mathcal{O}_{N}$ is always a free $\mathbf{Z}[G]$-module; this is equivalent to the statement $2 \cdot \bar{\chi}^{P}\left(\mathcal{O}_{X}\right)=0$.

In this paper we establish a connection between the problem of the vanishing of the obstructions $\bar{\chi}^{P}\left(\mathcal{O}_{X}\right)$ for higher dimensional varieties, and the theory of cyclotomic ideal class groups. As we will explain below, our results indicate a close connection between a positive answer to the above question for all unramified Galois covers $X \rightarrow Y$ of prime order $p>\operatorname{dim}(Y)$ and the truth of Vandiver's conjecture for $p$. Vandiver's conjecture for the prime number $p$ is the statement that $p$ does not divide the class number $h_{p}^{+}=\# \mathrm{Cl}\left(\mathbf{Q}\left(\zeta_{p}+\zeta_{p}^{-1}\right)\right)$.

Date: May 4, 2005.

MSC: 11R, 19A, 14F, Key words: Galois cover, coherent cohomology, ideal class group.

*Partially supported by NSF grants \# DMS05-01049 and \# DMS01-11298 (via the Institute for Advanced Study). 
This has been verified numerically for all $p<12 \cdot 10^{6}$ [BCEM]. However, there is doubt about its truth in general (see [W], p. 158). We show:

Theorem 1.1. Assume $G=\mathbf{Z} / p \mathbf{Z}$, with $p$ a prime.

a) If $p>\operatorname{dim}(X)$ and $p$ satisfies Vandiver's conjecture then $\bar{\chi}^{P}\left(\mathcal{O}_{X}\right)=0$.

b) There is an integer $N$ that depends only ond such that $N \cdot \bar{\chi}^{P}\left(\mathcal{O}_{X}\right)=0$ for all $G$-torsors $X / Y$ as above with $\operatorname{dim}(X) \leq d+1$.

Notice that in this case, by Rim's theorem, $\mathrm{Cl}(\mathbf{Z}[G])=\mathrm{Cl}\left(\mathbf{Q}\left(\zeta_{p}\right)\right)$. For simplicity, we set in this introduction $C=\mathrm{Cl}\left(\mathbf{Q}\left(\zeta_{p}\right)\right)$. Denote by ${ }_{p} C$ (resp. $\left.C / p\right)$ the kernel (resp. the cokernel) of multiplication by $p$ on $C=\mathrm{Cl}\left(\mathbf{Q}\left(\zeta_{p}\right)\right)$. We use the superscript $(k)$ to denote the eigenspace of ${ }_{p} C$ or $C / p$ on which $\sigma_{a} \in \operatorname{Gal}\left(\mathbf{Q}\left(\zeta_{p}\right) / \mathbf{Q}\right), \sigma_{a}\left(\zeta_{p}\right)=\zeta_{p}^{a}$, acts via multiplication by $a^{k}$. Recall the classical "reflection" homomorphisms $([\mathrm{W}] \S 10.2)$

$$
R^{(i)}: \operatorname{Hom}\left((C / p)^{(1-i)}, \mathbf{Z} / p \mathbf{Z}\right) \rightarrow{ }_{p} C^{(i)} .
$$

Assume that $p>\operatorname{dim}(X)=\operatorname{dim}(Y)=d+1$. The crux of our proof of Theorem 1.1 is to produce elements $t_{i}(X / Y)$ in $\operatorname{Hom}\left((C / p)^{(1-i)}, \mathbf{Z} / p \mathbf{Z}\right), 1 \leq i \leq d+1$, such that

$$
\bar{\chi}^{P}\left(\mathcal{O}_{X}\right)=\sum_{i=1}^{d+1} R^{(i)}\left(t_{i}(X / Y)\right)
$$

in $C$. By class field theory, each element $t_{i}(X / Y)$ corresponds to an unramified $\mathbf{Z} / p \mathbf{Z}$ extension of $\mathbf{Q}\left(\zeta_{p}\right)$. This extension depends only on the cover of generic fibers $X_{\mathbf{Q}} \rightarrow Y_{\mathbf{Q}}$. We would like to think of the $t_{i}(X / Y)$ 's as equivariant characteristic classes of $X_{\mathbf{Q}}$ with its $G$ action; at least $t_{d+1}(X / Y)$ can be obtained from the cover $X_{\mathbf{Q}} \rightarrow Y_{\mathbf{Q}}$ in a very explicit manner (see Remark 5.7). The formula (1.1) then resembles the output of a hypothetical "refined" Riemann-Roch theorem without denominators. When $p$ satisfies Vandiver's conjecture, we have $R^{(i)}=0$. Hence, $\bar{\chi}^{P}\left(\mathcal{O}_{X}\right)=0$ which shows (a). Part (b) follows from (1.1) and bounds on the order of the eigenspaces $(C / p)^{(1-i)}, 1 \leq i \leq d+1$, due to Herbrand, Kurihara [Ku] and Soulé [So]. In fact, when $d \leq 3$, we can take $N=1$.

At the moment, we are lacking direct evidence for the following:

Conjecture 1.2. For a given odd prime $p$ and $0 \leq k \leq p-2$, the set of elements $\left\{t_{k+1}(X / Y)\right\}$, $X \rightarrow Y$ ranging over all $\mathbf{Z} / p \mathbf{Z}$-torsors with $Y$ integral, projective and flat of relative dimension $k$ over $\operatorname{Spec}(\mathbf{Z})$, generate the group $\operatorname{Hom}\left((C / p)^{(-k)}, \mathbf{Z} / p \mathbf{Z}\right)$.

Since $R^{(i)}$ is injective for $i$ odd ([W]), this statement has the following (possibly vacuous) implication: Suppose $p$ does not satisfy Vandiver's conjecture. Then there is a $\mathbf{Z} / p \mathbf{Z}$-torsor $\pi: X \rightarrow Y$ with $Y \rightarrow \operatorname{Spec}(\mathbf{Z})$ projective and flat of relative dimension $d<p-1$ such that $\bar{\chi}^{P}\left(\mathcal{O}_{X}\right) \neq 0$. The expectation expressed here is that Vandiver's conjecture relates to the existence of a hypothetical coherent "Lefschetz fixed point theorem" in this situation "over Z". Indeed, since in the case of a $G$-torsor $X$ there are no fixed points, such a theorem would give $\bar{\chi}^{P}\left(\mathcal{O}_{X}\right)=0$. (In general, such a hypothetical fixed point theorem would describe how to calculate equivariant Euler characteristics like $\bar{\chi}^{P}\left(\mathcal{O}_{X}\right)$ from the fixed points of the action. Let us remark here that when the base is a field there are several classical results along these lines starting with the work of Atiyah and Bott.) 
We actually obtain more precise results and also results for $G$-torsors for a general finite group $G$. These generalize the main results of [P1] from the case of arithmetic surfaces to varieties of arbitrary dimension. In order to state these, we now introduce various numbers which feature in the annihilators of the equivariant Euler characteristics. Recall that the $k$-th Bernoulli number $B_{k}$ is defined by the powers series identity: $t /\left(e^{t}-1\right)=\sum_{k=0}^{\infty} B_{k} t^{k} / k$ !. For a prime $p$, and $a=p^{k} \cdot a^{\prime} \in \mathbf{Z}_{>0}$ with $\operatorname{gcd}\left(a^{\prime}, p\right)=1$, we set $\operatorname{ord}_{p}(a)=p^{k}$. For $n \geq 2$, let us set

$$
e(n)= \begin{cases}\text { numerator }\left(B_{n} / n\right) & , \text { if } n \text { is even, } \\ \prod_{p, p \mid h_{p}^{+}} \operatorname{ord}_{p}\left(\# \mathrm{~K}_{2 n-2}(\mathbf{Z})\right) & , \text { if } n \text { is odd, }\end{cases}
$$

where $\mathrm{K}_{2 n-2}(\mathbf{Z})$ is the Quillen K-group (by work of Borel [Bo], this is a finite group for $n>1)$. We set

$$
M_{n}(G)=\prod_{k=2}^{n}\left(\prod_{p \mid e(k)} \operatorname{ord}_{p}(\# G)\right), \quad M_{n}^{\prime}(G)=\prod_{s=1}^{[n / 2]}\left(\prod_{p \mid e(2 s)} \operatorname{ord}_{p}(\# G)\right) .
$$

We have $M_{n}(G)=1$ for $n \leq 4$ and all $G, M_{n}^{\prime}(G)=1$ for $n \leq 11$ and all $G$. In general, if \#G is relatively prime to $e(k)$ for all $2 \leq k \leq n$, then $M_{n}(G)=1$.

Now let $\mathcal{M}_{G}$ be a maximal $\mathbf{Z}$-order in $\mathbf{Q}[G]$ which contains $\mathbf{Z}[G]$ and denote by $\operatorname{Cl}\left(\mathcal{M}_{G}\right)$ the class group of finitely generated projective $\mathcal{M}_{G}$-modules. Let us write $\mathbf{Q}[G] \simeq \prod_{i} M_{n_{i}}\left(D_{i}\right)$ with $D_{i}$ a division algebra. By a result of Eichler, if $R_{i}$ is the ring of integers of the center $C_{i}$ of $D_{i}$, then

$$
\mathrm{Cl}\left(\mathcal{M}_{G}\right) \simeq \prod_{i} \mathrm{Cl}^{+}\left(R_{i}\right)
$$

where $\mathrm{Cl}^{+}\left(R_{i}\right)$ is the class group of $R_{i}$-ideals modulo principal ideals which are generated by elements which are positive at each infinite place of $C_{i}$ where $D_{i}$ is a quaternionic division algebra ([F] I §2). Tensoring with $\mathcal{M}_{G}$ over $\mathbf{Z}[G]$ induces a group homomorphism

$$
\Phi: \mathrm{Cl}(\mathbf{Z}[G]) \rightarrow \mathrm{Cl}\left(\mathcal{M}_{G}\right) \simeq \prod_{i} \mathrm{Cl}^{+}\left(R_{i}\right) .
$$

In the following statements, $\pi: X \rightarrow Y$ is a $G$-torsor with $Y \rightarrow \operatorname{Spec}(\mathbf{Z})$ projective and flat of relative dimension $d$ and $\mathcal{F}$ is a $G$-equivariant coherent sheaf on $X$.

Theorem 1.3. a) For all finite groups $G$, we have

$$
M_{d+1}(G) \cdot \Phi\left(\bar{\chi}^{P}(\mathcal{F})\right)=0 .
$$

b) Assume that all the Sylow subgroups of the group $G$ are abelian. Then

$$
\operatorname{gcd}(2, \# G) M_{d+1}(G) \cdot \bar{\chi}^{P}(\mathcal{F})=0 .
$$

Theorem 1.4. Assume that for all the prime divisors $p$ of the order $\# G$, the eigenspaces $(C / p)^{(k)}$ for all even $k$ in $[1, d+1] \cup[p-1-d, p-1]$ are trivial. (This is true if $p$ satisfies Vandiver's conjecture.) Set $C_{d+1}(G)=\operatorname{gcd}\left(M_{d+1}^{\prime}(G), 2((d+1) ! !)\right)$ with $(d+1) ! !=(d+$ $1) ! d ! \cdots 2$ !. Then

$$
C_{d+1}(G) \cdot \Phi\left(\bar{\chi}^{P}(\mathcal{F})\right)=0 .
$$

In particular, if all the prime divisors of \#G satisfy Vandiver's conjecture and are larger than $d+1$ then $\Phi\left(\bar{\chi}^{P}(\mathcal{F})\right)=0$. 
Notice that Theorem 1.1 follows from these results and Rim's theorem ([Ri]): For $G=$ $\mathbf{Z} / p \mathbf{Z}, p$ prime, $\Phi$ is an isomorphism.

We also obtain:

Theorem 1.5. Suppose that $G$ is abelian.

a) If $\pi: X \rightarrow Y$ is of Albanese type (see $\S 5 . c)$ then $\bar{\chi}^{P}(\mathcal{F})$ is trivial in $\mathrm{Cl}(\mathbf{Z}[G])$.

b) If $\pi: X_{\mathbf{Q}} \rightarrow Y_{\mathbf{Q}}$ is of Albanese type then the multiple $2((d+1) ! !) \cdot \bar{\chi}^{P}(\mathcal{F})$ is trivial in $\mathrm{Cl}(\mathbf{Z}[G])$.

If $Y_{\mathbf{Q}}$ is smooth, has a rational point and $X_{\mathbf{Q}}$ is geometrically connected, the condition that $X_{\mathbf{Q}} \rightarrow Y_{\mathbf{Q}}$ is of Albanese type means that the cover is obtained by specializing an isogeny of the Albanese variety of $Y_{\mathbf{Q}}$.

Let us now discuss the proof of these results. Determining the classes $\bar{\chi}^{P}(\mathcal{F})$ is a subtle problem. Indeed, these lie in the finite group $\mathrm{Cl}(\mathbf{Z}[G])$ and cannot be calculated using the known "Riemann-Roch type" theorems that usually neglect torsion information. Instead, our basic tool is the notion of " $n$-cubic structure" on line bundles over commutative group schemes which was introduced and studied in [P2]. This is a generalization of the notion of cubic structure (for $n=3$ ) of Breen $[\mathrm{Br}]$. The starting point for us is the fact, shown by F. Ducrot $[\mathrm{Du}]$, that the determinant of cohomology along a projective and flat morphism $Y \rightarrow S$ of relative dimension $d$ essentially supports a $d+2$-cubic structure (see $\S 4$ ). When $G$ is abelian, we deduce that the square of the determinant $\operatorname{det}_{\mathbf{Z}[G]} \mathbf{R} \Gamma\left(X, \mathcal{O}_{X}\right)^{\otimes 2}$ gives a line bundle over $G^{D}:=\operatorname{Spec}(\mathbf{Z}[G])$ which supports a $d+2$-cubic structure. We then use the results of [P2] on line bundles with $n$-cubic structures over multiplicative groups like $G^{D}$. In loc. cit. the group of line bundles with $n$-cubic structures is studied inductively using "multiextensions". When $G=\mathbf{Z} / p \mathbf{Z}$, one sees that a $k$-multiextension over $G^{D}=\mu_{p}$ is given by an unramified $\mathbf{Z} / p \mathbf{Z}$-extension $L$ of $\mathbf{Q}\left(\zeta_{p}\right)$ such that $\sigma_{a} \in \operatorname{Gal}\left(\mathbf{Q}\left(\zeta_{p}\right) / \mathbf{Q}\right)$ acts by conjugation on $\mathbf{Z} / p \mathbf{Z}=\operatorname{Gal}\left(L / \mathbf{Q}\left(\zeta_{p}\right)\right)$ via multiplication by $a^{1-k}$. In fact, the elements $t_{i}(X / Y), 1 \leq i \leq d+1$, mentioned above, correspond to $i$-multiextensions associated naturally to the $d+2$-cubic structure on $\operatorname{det}_{\mathbf{Z}[G]} \mathbf{R} \Gamma\left(X, \mathcal{O}_{X}\right)^{\otimes 2}$. In general, the group of multiextensions over $G^{D}$ can always be described using eigenspaces of cyclotomic ideal class groups; hence we can bound the support of the group of multiextensions using results on the corresponding eigenspaces of these class groups. This technique applies directly when $G$ is abelian and to $\mathcal{F}=\mathcal{O}_{X}$; the case of more general finite groups and general $\mathcal{F}$ follows from these results using Noetherian induction and "localization" as in [P1].

The methods of this paper generalize to various other situations. For example, it is possible to treat torsors for a general finite and flat commutative group scheme over a Dedekind ring; then the annihilators of Euler characteristics will depend on the arithmetic of the fields involved. Also, our technique provides with the most precise general method for determining the Galois module structure of abelian covers even when we allow some (tame) ramification. Then one can not expect $\bar{\chi}^{P}(\mathcal{F})$ to vanish. However, the theory of this paper combined with the Grothendieck-Riemann-Roch theorem implies "fixed point formulas" which, for tame and abelian covers, calculate (up to an error annihilated by $2 \cdot M_{d+1}(G)$ ) the classes $\bar{\chi}^{P}(\mathcal{F})$ via localization on the ramification locus. This has since been developed in joint work with T. Chinburg and M. Taylor [CPT]. 
The author would like to thank T. Chinburg, R. Kulkarni, M. Rost, M. Taylor, and L. Washington for useful conversations, and F. Ducrot for making available a preliminary version of $[\mathrm{Du}]$.

\section{Preliminaries}

2.a. Let $B$ be a left Noetherian associative ring with unit. Most of the time we will take $B$ to be the group ring $R[G]$ of a finite group $G$ with $R$ commutative Noetherian. We denote by $\mathrm{K}_{0}(B)$ the Grothendieck group of finitely generated projective left $B$-modules, and by $\mathrm{K}_{0}^{\mathrm{red}}(B)$ the quotient of $\mathrm{K}_{0}(B)$ by the subgroup generated by the class $[B]$ of the free $B$ module $B$. We will denote by $a^{\text {red }}$ the image of the element $a$ of $\mathrm{K}_{0}(B)$ in $\mathrm{K}_{0}^{\mathrm{red}}(B)$. If $B$ is commutative, we will denote by $\operatorname{Pic}(B)$ the Picard group of $B$; then a finitely generated $B$-module is projective if and only if it is locally free. Taking highest exterior powers of locally free $B$-modules defines in this case a group homomorphism

$$
i: \mathrm{K}_{0}^{\mathrm{red}}(B) \rightarrow \operatorname{Pic}(B) .
$$

If $B$ is commutative and of Krull dimension 1, then by [BM] Cor. 3.5, the map $i$ is an isomorphism and we will use it to identify $\mathrm{K}_{0}^{\mathrm{red}}(B)$ with $\operatorname{Pic}(B)$.

We will denote by $D^{+}(B)$ the derived category of the homotopy category of complexes of left $B$-modules which are bounded below. Recall that a complex $C^{\bullet}$ in $D^{+}(B)$ is called "perfect", if it is isomorphic in $D^{+}(B)$ to a bounded complex $P^{\bullet}$ of finitely generated projective left $B$-modules. Then the element

$$
\chi\left(C^{\bullet}\right)=\sum_{i}(-1)^{i}\left[P^{i}\right] \in \mathrm{K}_{0}(B)
$$

depends only on the isomorphism class of $C^{\bullet}$ in $D^{+}(B)$.

For a scheme $Y$, we will denote by $\mathrm{G}_{0}(Y)$, resp. $\mathrm{K}_{0}(Y)$, the Grothendieck group of coherent,

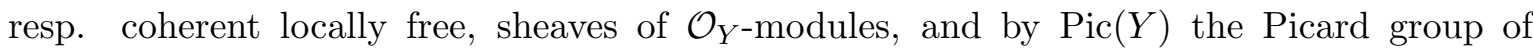
$Y$. If $Y=\operatorname{Spec}(B)$ is affine, we will identify quasi-coherent sheaves of $\mathcal{O}_{Y}$-modules on $Y$ with $B$-modules. This gives natural identifications $\mathrm{K}_{0}(B)=\mathrm{K}_{0}(\operatorname{Spec}(B))$ and $\operatorname{Pic}(B)=$ $\operatorname{Pic}(\operatorname{Spec}(B))$.

2.b. We refer to [SGA4] XVIII, 1.4. for the notion of a (commutative) Picard category. By definition, this is a non-empty category $\mathcal{P}$ in which all morphisms are isomorphisms and which is equipped with an "addition" functor $+: \mathcal{P} \times \mathcal{P} \rightarrow \mathcal{P}, \quad\left(p_{1}, p_{2}\right) \mapsto p_{1}+p_{2}$, associativity isomorphisms $\sigma_{p_{1}, p_{2}, p_{3}}:\left(p_{1}+p_{2}\right)+p_{3} \stackrel{\sim}{\rightarrow} p_{1}+\left(p_{2}+p_{3}\right)$, functorial in $p_{1}, p_{2}, p_{3}$, and commutativity isomorphisms $\tau_{p_{1}, p_{2}}: p_{1}+p_{1} \stackrel{\sim}{\longrightarrow} p_{2}+p_{1}$, functorial in $p_{1}, p_{2}$, which satisfy the axioms described in loc. cit. A Picard category is always equipped with an "identity object"; this is a pair $(\underline{O}, \epsilon)$ of an object $\underline{O}$ with an isomorphism $\epsilon: \underline{O}+\underline{O} \stackrel{\sim}{\rightarrow} \underline{O}$ which is unique up to unique isomorphism. If we have $\tau_{p, p}=\operatorname{Id}_{p+p}$, for all objects $p$ of $\mathcal{P}$, then we say that the Picard category is "strictly commutative" (s.c.).

Note that a commutative group defines a "discrete" s.c. Picard category: The objects are the elements of the group, the only morphisms are the identity morphisms and the addition is given by the group law. We will also often use the Picard categories $\operatorname{PIC}(S)$, resp. $\operatorname{PIC}_{*}(S)$, 
of invertible $\mathcal{O}_{S}$-sheaves, resp. "graded" invertible $\mathcal{O}_{S}$-sheaves over a scheme $S$ (see [De]). The Picard category $\operatorname{PIC}(S)$ is strictly commutative but $\mathrm{PIC}_{*}(S)$ is not.

If $\mathcal{P}$ and $\mathcal{Q}$ are two Picard categories an additive functor $F: \mathcal{P} \rightarrow \mathcal{Q}$ is a functor equipped with isomorphisms $f_{p_{1}, p_{2}}: F\left(p_{1}\right)+F\left(p_{2}\right) \stackrel{\sim}{\longrightarrow} F\left(p_{1}+p_{2}\right)$ for all objects $p_{1}, p_{2}$ of $\mathcal{P}$ which are functorial in $p_{1}, p_{2}$ and which are appropriately compatible with the associativity and commutativity isomorphisms of $\mathcal{P}$ and $\mathcal{Q}$ (see [SGA4] XVIII.)

We refer the reader to [SGA4] XVIII 1.4 for the definitions of a (s.c.) Picard stack over a site $\mathfrak{S}$ and of an additive (్-)functor between two Picard $\mathfrak{S}$-stacks (these are modeled on the definitions above). We will mainly work with the site $S_{\mathrm{fppf}}$ of $S$-schemes which are locally of finite presentation with the fppf topology. In this case, we may think of a Picard $S_{\mathrm{fppf}}$-stack as a fibered category such that the fiber over each $S^{\prime} \rightarrow S$ is a Picard category, with additive pull-back functors and which satisfies a certain descent condition for both the objects and the morphisms. As before, a commutative group scheme over $S$ gives a "discrete" fppf s.c. Picard stack. If $\phi: T \rightarrow S$ is an $S$-scheme we will denote by $\mathcal{P} \mathcal{I C}(T)$ (resp. $\mathcal{P} \mathcal{I C} \mathcal{C}_{*}(T)$ ) the

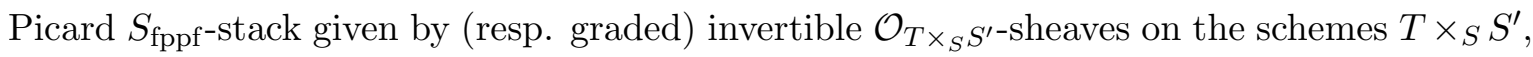
$S^{\prime} \rightarrow S$ a morphism in $S_{\text {fppf }}$.

2.c. Let $H \rightarrow S$ be an $S$-group scheme flat and affine over $S$. We refer to [DG] III for the notion of an $H$-torsor. Under our assumptions, this is given by a scheme $p: T \rightarrow S$ with right action of $H$ and $p$ affine faithfully flat such that the map $T \times_{S} H \rightarrow T \times_{S} T$ given by $(t, h) \mapsto(t \cdot h, t)$ is an isomorphism. If $Y$ is an $S$-scheme we will use the expression " $X \rightarrow Y$ is an $H$-torsor" to mean that $X \rightarrow Y$ is a torsor for the group scheme $H_{Y}:=H \times_{S} Y \rightarrow Y$. If $\pi: X \rightarrow Y$ is an $H$-torsor, then $\pi$ is affine and faithfully flat and identifies $Y$ with the (categorical) quotient $X / H$. In fact, this quotient is universal in the sense that for every base change $S^{\prime} \rightarrow S$, the natural morphism $\left(X \times_{S} S^{\prime}\right) / H \rightarrow(X / H) \times_{S} S^{\prime}$ is an isomorphism. If $H \rightarrow S$ is in addition of finite presentation then so is $\pi: X \rightarrow Y$.

If $H \rightarrow S$ is commutative then the category of $H$-torsors over $S$ has a natural addition functor which makes it a s.c. Picard category (see [DG] III §4.) Denote by $\mathbf{G}_{m}$ the multiplicative group scheme over $S$. There is a natural additive equivalence between the category PIC(T) of invertible $\mathcal{O}_{T}$-sheaves over an $S$-scheme $T$ and the category of $\mathbf{G}_{m T}$-torsors on $T$ given by $\mathcal{L} \rightarrow \underline{\operatorname{Isom}}_{\mathcal{O}_{T}}\left(\mathcal{O}_{T}, \mathcal{L}\right)$. (In what follows, we will denote the $\mathbf{G}_{m T}$-torsor associated to the invertible sheaf $\mathcal{L}$ again by $\mathcal{L})$.

2.d. Assume now that $G$ is a finite group; for a scheme $S$ we will denote by $G_{S}$ the constant group scheme $\sqcup_{g \in G} S$ given by $G$. When $S=\operatorname{Spec}(\mathbf{Z})$ we will abuse notation and simply write $G$ instead of $G_{S}$. Let $T$ be an $S$-scheme with a right $G$-action (this is the same as a right $G_{S}$-action). We will say that $G$ acts freely on $T$ if for every $S$-scheme $S^{\prime}$ the action of $G$ on the set of $T\left(S^{\prime}\right)$ of $S^{\prime}$-points of the $S$-scheme $T$ is free (i.e all the stabilizers are trivial). If $T \rightarrow S$ is quasi-projective, then the quotient $T / G$ exists as a scheme; when in addition $G$ acts freely on $T$ then $\pi: T \rightarrow T / G$ is a $G$-torsor and the morphism $\pi$ is finite étale ([D-G] III, $\left.\S 2, n^{\circ} 6\right)$. We will assume this is the case in the rest of this paragraph.

A coherent (resp. locally free coherent) sheaf of $\mathcal{O}_{T}-G$-modules $\mathcal{F}$ on $T$ is a coherent (resp. locally free coherent) sheaf of $\mathcal{O}_{T}$-modules with an action of $G$ compatible with the action of $G$ on $\left(T, \mathcal{O}_{T}\right)$ in the appropriate sense. We will often use the term $G$-equivariant coherent 
(resp. $G$-equivariant locally free coherent) sheaf on $T$ instead of coherent (resp. locally free coherent) $\mathcal{O}_{T}$-G-sheaf. Let $\mathcal{F}$ be a $G$-equivariant coherent sheaf on $T$ and suppose that $V=\operatorname{Spec}(C)$ is an open affine subscheme of $T / G$. Since $\pi$ is finite, $U=\pi^{-1}(V)$ is an affine $G$-equivariant open subscheme of $T$. The sections $\mathcal{F}(U)$ form a left $G$-module and since $\mathcal{F}(U)$ is also a $C$-module, we obtain on it the structure of a left $C[G]$-module. Hence, $\mathcal{F}$ provides us with a coherent sheaf of $\mathcal{O}_{T / G}[G]$-modules on $T / G$, which we will denote by $\pi_{*}(\mathcal{F})$. On the other hand, if $\mathcal{G}$ is a coherent (locally free coherent) sheaf of $\mathcal{O}_{T / G}$-modules on $T / G$, the pull back $\pi^{*}(\mathcal{G})$ is a $G$-equivariant coherent (resp. locally free coherent) sheaf on $T$. By descent, the pull-back functor $\pi^{*}$ gives an equivalence between the category of coherent (resp. locally free coherent) sheaves on the quotient $T / G$ and the category of $G$-equivariant coherent (resp. locally free coherent) sheaves on $T$. Its inverse functor is obtained by taking invariants under $G$ of the direct image $\pi_{*}$.

2.e. Now suppose that in addition $G$ is commutative. Denote by $G_{S}^{D}$ the Cartier dual group scheme of $G_{S}$; by definition, this represents the sheaf of characters $\underline{\operatorname{Hom}}\left(G_{S}, \mathbf{G}_{m S}\right)$. Let $\chi: G \rightarrow \Gamma\left(S^{\prime}, \mathcal{O}_{S^{\prime}}^{*}\right)=\mathbf{G}_{m}\left(S^{\prime}\right)$ be a character with $S^{\prime} \rightarrow S$ an $S$-scheme; then $\chi$ corresponds to an $S^{\prime}$-point of $G_{S}^{D}$. Let $Y \rightarrow S$ be an $S$-scheme and $\pi: X \rightarrow Y$ a $G$-torsor; we will denote by $\pi^{\prime}: X^{\prime}=X \times{ }_{S} S^{\prime} \rightarrow Y^{\prime}=Y \times{ }_{S} S^{\prime}$ the base change. Now we can use the character $\chi$ to define the structure of an $\mathcal{O}_{Y^{\prime}}-G$-module $\mathcal{O}_{X^{\prime}}(\chi)$ on $\mathcal{O}_{X^{\prime}}$ by setting $g \cdot\left(m \otimes a^{\prime}\right)=g \cdot m \otimes \chi(g)^{-1} a^{\prime}$, where $m, a^{\prime}$ stand for sections of $\mathcal{O}_{X}$, resp. $\mathcal{O}_{S^{\prime}}$. Taking the $G$-invariants $\left(\pi_{*}^{\prime}\left(\mathcal{O}_{X^{\prime}}(\chi)\right)\right)^{G}$ of the direct image by $\pi^{\prime}$ gives an invertible sheaf on $Y^{\prime}$ which we will denote by $\mathcal{O}_{X, \chi}$. We obtain a functor $\chi \mapsto \mathcal{O}_{X, \chi}$ from the discrete Picard category $G^{D}\left(S^{\prime}\right)$ to the s.c. Picard category $\operatorname{PIC}\left(Y^{\prime}\right)$. Notice that the invertible sheaf $\mathcal{O}_{X, \chi}$ corresponds to the (étale) $\mathbf{G}_{m^{-}}$ torsor over $S^{\prime}$ which is obtained from the $G$-torsor $\pi^{\prime}$ by changing the structure group using $\chi^{-1}: G \rightarrow \mathbf{G}_{m S^{\prime}}$.

Recall that $\pi_{*}\left(\mathcal{O}_{X}\right)$ has the structure of a coherent sheaf of $\mathcal{O}_{Y}[G]$-modules on $Y$. Hence, we may think of $\pi_{*}\left(\mathcal{O}_{X}\right)$ as a coherent $\mathcal{O}_{G_{Y}^{D}}$-sheaf on $G_{Y}^{D}=Y \times_{S} G_{S}^{D}$. Suppose now that $\chi_{0}: G \rightarrow \mathbf{G}_{m}\left(G_{S}^{D}\right)$ is the "universal" $G_{S}^{D}$-valued character obtained from the natural pairing $G_{S}^{D} \times{ }_{S} G_{S} \rightarrow \mathbf{G}_{m S}$. We have

$$
\mathcal{O}_{X, \chi_{0}} \simeq\left(\pi_{*}\left(\mathcal{O}_{X}\right) \otimes_{\mathcal{O}_{Y}} \mathcal{O}_{G_{Y}^{D}}\right)^{G}=\left(\pi_{*}\left(\mathcal{O}_{X}\right)[G]\right)^{G}
$$

with the $G$-action defined by $g \cdot\left(\sum_{h} a_{h} h\right)=\sum_{h} g\left(a_{h}\right) g^{-1} h$. We can see that this gives an isomorphism

$$
\mathcal{O}_{X, \chi_{0}} \simeq \pi_{*}\left(\mathcal{O}_{X}\right)
$$

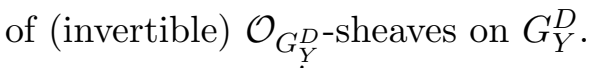

If $\chi_{1}, \chi_{2}$ are $S^{\prime}$-valued characters of $G$ as above, the multiplication in $\mathcal{O}_{X^{\prime}}$ induces a $G$ equivariant isomorphism $\mathcal{O}_{X^{\prime}}\left(\chi_{1}\right) \otimes_{\mathcal{O}_{X^{\prime}}} \mathcal{O}_{X^{\prime}}\left(\chi_{2}\right) \stackrel{\sim}{\longrightarrow} \mathcal{O}_{X^{\prime}}\left(\chi_{1} \chi_{2}\right)$. By descent (§2.d) this gives an isomorphism of invertible $\mathcal{O}_{Y^{\prime} \text {-sheaves }}$

$$
c_{\chi_{1}, \chi_{2}}: \mathcal{O}_{X, \chi_{1}} \otimes_{\mathcal{O}_{Y^{\prime}}} \mathcal{O}_{X, \chi_{2}} \stackrel{\sim}{\longrightarrow} \mathcal{O}_{X, \chi_{1} \chi_{2}} .
$$

We can see that the isomorphisms $c_{\chi_{1}, \chi_{2}}$ are compatible with the associativity and commutativity constraints of the source and target commutative Picard categories. Hence, they equip 
the functor $\chi \mapsto \mathcal{O}_{X, \chi}$ with additivity data. In fact, using the fact that the construction is compatible with base change we see that we obtain an additive functor

$$
F_{\pi}: G_{S}^{D} \rightarrow \mathcal{P} \mathcal{I C}(Y) ; \quad F_{\pi}(\chi)=\mathcal{O}_{X, \chi}
$$

between Picard $S_{\text {fppf }}$-stacks.

Remark 2.1. As explained in [SGA4] XVIII 1.4 the additive functor $F_{\pi}$ gives a commutative extension of groups schemes over $Y$

$$
1 \rightarrow \mathbf{G}_{m Y} \rightarrow E \rightarrow G_{Y}^{D} \rightarrow 1
$$

such that the fiber of $E$ over the $Y^{\prime}=Y \times_{S} S^{\prime}$-point of $G_{Y}^{D}$ which is given by the character $\chi$ is isomorphic to the $\mathbf{G}_{m Y^{\prime}}$-torsor that corresponds to the invertible $\mathcal{O}_{Y^{\prime}}$-sheaf $\mathcal{O}_{X^{\prime}, \chi}$ (see also [SGA7I] VII). Conversely, a commutative extension of $G_{Y}^{D}$ by $\mathbf{G}_{m Y}$ defines an additive functor $G_{S}^{D} \rightarrow \mathcal{P} \mathcal{I C}(Y)$; if two extensions are isomorphic, the corresponding additive functors are isomorphic (as additive functors; compare to [SGA4] XVIII 1.4.23).

\section{Hypercubic StRuCtures AND MUltieXtensions}

In this section we recall the definition of " $n$-cubic structure" and some of the main results of [P2]. The reader can refer to [P2] for more details.

3.a. Let $H \rightarrow S$ be a commutative $S$-group scheme. For $n \geq 1$, we will denote by $H^{n}:=$ $H \times \cdots \times H$ the $n$-fold fiber product over $S$. If $I$ is a subset of the index set $\{1, \ldots, n\}$, we will denote by $m_{I}$ the morphism $H^{n} \rightarrow H$ given on points by $\left(h_{1}, \ldots, h_{n}\right) \mapsto \sum_{i \in I} h_{i}$ (if $\left.I=\emptyset, m_{I}\left(h_{1}, \ldots, h_{n}\right)=0\right)$. Recall that we identify the s.c. Picard category of invertible sheaves over an $S$-scheme $T$ with the s.c. Picard category of $\mathbf{G}_{m T}$-torsors on $T$ (see 2.c).

If $\mathcal{L}$ is an invertible sheaf on $H$, then we set

$$
\Theta_{n}(\mathcal{L})=\bigotimes_{I \subset\{1, \ldots, n\}} m_{I}^{*}(\mathcal{L})^{(-1)^{n-\# I}}
$$

(an invertible sheaf on $H^{n}$ ). A permutation $\sigma:\{1, \ldots, n\} \rightarrow\{1, \ldots, n\}$ induces a corresponding $S$-isomorphism $\sigma: H^{n} \rightarrow H^{n}$. Since $m_{I} \cdot \sigma=m_{\sigma(I)}$, permuting the factors of the tensor product gives a canonical isomorphism

$$
\mathfrak{P}_{\sigma}: \sigma^{*} \Theta_{n}(\mathcal{L}) \stackrel{\sim}{\longrightarrow} \Theta_{n}(\mathcal{L}) .
$$

Now suppose that $n \geq 2$ and consider the morphisms $A, B, C, D: H^{n+1} \rightarrow H^{n}$ given by

$$
\begin{aligned}
& A\left(h_{0}, h_{1}, h_{2}, \ldots, h_{n}\right)=\left(h_{0}+h_{1}, h_{2}, \ldots, h_{n}\right), \\
& B\left(h_{0}, h_{1}, h_{2}, \ldots, h_{n}\right)=\left(h_{0}, h_{1}, h_{3}, \ldots, h_{n}\right), \\
& C\left(h_{0}, h_{1}, h_{2}, \ldots, h_{n}\right)=\left(h_{0}, h_{1}+h_{2}, h_{3}, \ldots, h_{n}\right), \\
& D\left(h_{0}, h_{1}, h_{2}, \ldots, h_{n}\right)=\left(h_{1}, h_{2}, h_{3}, \ldots, h_{n}\right) .
\end{aligned}
$$

We can observe that there is a canonical isomorphism

$$
\mathfrak{Q}: A^{*} \Theta_{n}(\mathcal{L}) \otimes B^{*} \Theta_{n}(\mathcal{L}) \stackrel{\sim}{\longrightarrow} C^{*} \Theta_{n}(\mathcal{L}) \otimes D^{*} \Theta_{n}(\mathcal{L})
$$


which is obtained by contracting duals and permuting factors (The order in which these operations are performed in the s.c. Picard category is of no consequence; the isomorphism remains the same.)

Finally observe that if $(0, \ldots, 0): S \rightarrow H^{n}$ is the zero section, there is a canonical isomorphism

$$
\mathfrak{R}:(0, \ldots, 0)^{*} \Theta_{n}(\mathcal{L}) \stackrel{\sim}{\longrightarrow} \mathcal{O}_{S} .
$$

Definition 3.1. Let $n \geq 2$. An $n$-cubic structure on the invertible sheaf $\mathcal{L}$ over $H$ is an isomorphism of invertible sheaves on $H^{n}$

$$
\xi: \mathcal{O}_{H^{n}} \stackrel{\sim}{\longrightarrow} \Theta_{n}(\mathcal{L})
$$

(i.e a choice of a global generator $\xi(1)$ of $\Theta_{n}(\mathcal{L})$ ) which satisfies the following conditions:

c0) It is "rigid", i.e we have $\mathfrak{R}\left((0, \ldots, 0)^{*}(\xi(1))\right)=1$.

c1) It is "symmetric", i.e for all $\sigma \in S_{n}, \mathfrak{P}_{\sigma}\left(\sigma^{*}(\xi(1))\right)=\xi(1)$.

c2) It satisfies the "cocycle condition"

$$
\mathfrak{Q}\left(A^{*}(\xi(1)) \otimes B^{*}(\xi(1))\right)=C^{*}(\xi(1)) \otimes D^{*}(\xi(1)) .
$$

3.b. Suppose that $J$ and $H$ are two flat commutative group schemes over the scheme $S$. We will assume that $J \rightarrow S$ is affine. (Most of the time we will take $J=\mathbf{G}_{m}$ and $H=G_{S}^{D}$.) We refer the reader to [SGAI] Exposé VII, Remark 3.6.7 (or [P2] §4) for the definition of a $n$-extension of $(H, \ldots, H)$ by $J$. (For simplicity, we will just say " $n$-extension of $H$ by $J$ ". For $n=1$ this gives a usual extension of commutative group schemes and for $n=2$ a $J$-biextension of $(H, H)$ as in [SGA7I] Exposé VII.) By definition, such an $n$-extension is a $J$-torsor $E$ over $H^{n}$ equipped with "compatible partial composition laws". We will denote by $n$ - $\operatorname{Ext}^{1}(H, J)$ the commutative group of isomorphism classes of $n$-extensions of $H$ by $J$. Note that sending the class of an $n$-extension to the class of the underlying $J$-torsor over $H^{n}$ defines a group homomorphism

$$
t: n-\operatorname{Ext}^{1}(H, J) \rightarrow \mathrm{H}^{1}\left(H^{n}, J\right) .
$$

When $J=\mathbf{G}_{m}$, this is a homomorphism $t: n-\operatorname{Ext}^{1}\left(H, \mathbf{G}_{m}\right) \rightarrow \operatorname{Pic}\left(H^{n}\right)$.

3.c. Here, for the convenience of the reader, we recall the main results of [P2] (loc. cit. Theorems 1.1 and 8.4). The numbers $e(k), M_{n}(G), M_{n}^{\prime}(G), \operatorname{ord}_{p}$ are as defined in the introduction and we set $H=G_{\text {Spec }(\mathbf{z})}^{D}$ where $G$ is abelian.

Theorem 3.2. The group of isomorphism classes of line bundles with n-cubic structure $(\mathcal{L}, \xi)$ over $H$ is annihilated by

$$
M=M_{n-1}(G)=\prod_{k=1}^{n-1} \prod_{p, p \mid e(k)} \operatorname{ord}_{p}(\# G) .
$$

In particular, if $\mathcal{L}$ is a line bundle over $H$ which supports an $n$-cubic structure then we have $\mathcal{L}^{\otimes M} \simeq \mathcal{O}_{H}$.

Notice that since $B_{2}=1 / 6, B_{4}=-1 / 30$ and $\mathrm{K}_{4}(\mathbf{Z})$ is trivial $([\operatorname{Ro}])$, we have $M_{n-1}(G)=1$ for $n \leq 5$ and all $G$. 
Theorem 3.3. Suppose that $\mathcal{L}$ is an invertible sheaf on $H$ which supports an $n$-cubic structure. We denote by $\nu: \widetilde{H} \rightarrow H$ the normalization morphism. Assume that for all the prime divisors $p$ of the order $\# G$, the eigenspaces $\left(\mathrm{Cl}\left(\mathbf{Q}\left(\zeta_{p}\right)\right) / p\right)^{(k)}$ for all even $k$ in $[1, n-1] \cup$ $[p-n+1, p-1]$ are trivial. (This is true if $p$ satisfies Vandiver's conjecture.) Let us set $C=\operatorname{GCD}\left(M_{n-1}^{\prime}(G),(n-1) ! !\right)$. Then $\nu^{*} \mathcal{L}^{\otimes C} \simeq \mathcal{O}_{\widetilde{H}}$. In particular, if in addition all the prime divisors of $\# G$ are $\geq n$, then $\nu^{*} \mathcal{L} \simeq \mathcal{O}_{\widetilde{H}}$.

\section{The hypercubic Structure on the Determinant of COHOMOlOGY}

In this section, we explain some of the results of F. Ducrot $[\mathrm{Du}]$ and we show how the work in $[\mathrm{Du}]$ can be used to deduce the main result in this section, Theorem 4.7.

For every non-empty finite set $I$ we denote by $C(I)$ the set of all subsets of $I$. Suppose that $\mathcal{P}$ is a s.c. Picard category (§2.b). By definition, an $I$-cube in $\mathcal{P}$ is a family $K=\left(K_{v}\right)_{v \in C(I)}$ of objects of $\mathcal{P}$, indexed by the set $C(I)$ of all subsets of $I$. We denote by $I-C u b e(\mathcal{P})$ the category of $I$-cubes in $\mathcal{P}$.

If $n \geq 1$, then an $n$-cube in $\mathcal{P}$ is by definition an $I$-cube in $\mathcal{P}$ for some $I$ with $\# I=n$. A morphism between two $n$-cubes $K=\left(K_{v}\right)_{v \in C(I)}, K^{\prime}=\left(K_{w}^{\prime}\right)_{w \in C\left(I^{\prime}\right)}$ is a pair $\Phi=(\varphi, \phi)$ where $\varphi: I \stackrel{\sim}{\longrightarrow} I^{\prime}$ is bijective and $\phi: K \stackrel{\sim}{\longrightarrow} \varphi^{*}\left(K^{\prime}\right)$ is an (iso)morphism of $I$-cubes. The category of all $n$-cubes in $\mathcal{P}$ will be denoted by $n$-Cube $(\mathcal{P})$.

This "cubic" terminology is motivated by the fact that if $I=\{1,2, \ldots, n\}$ we may think of $C(I)$ as the vertices of the standard $n$-dimensional cube $C_{n}$ in $\mathbf{R}^{n}$ by sending a subset $v \subset I$ to the point $\left(v_{i}\right)_{1 \leq i \leq n}$ with $v_{i}=1$ if $i \in v, v_{i}=0$ if $i \notin v$. Hence, after choosing an order for $I$, we can visualize the objects $K_{v}$ of an $I$-cube $K$ as being placed on the vertices of the $n$-dimensional cube $C_{n}$.

For $v \in C(I)$, set $s(v)=(-1)^{\# I-\# v}$. If $K$ is an $n$-cube in $\mathcal{P}$ we now set

$$
\Sigma(K)=\sum_{v \in C(I)}(-1)^{s(v)} K_{v},
$$

where $-K$ denotes the negative (or "inverse") of the object $K$ in $\mathcal{P}$ (see [De]). We can see that an isomorphism $\Phi: K \rightarrow K^{\prime}$ between $n$-cubes induces an isomorphism

$$
\Sigma(\Phi): \Sigma(K) \stackrel{\sim}{\longrightarrow} \Sigma\left(K^{\prime}\right)
$$

in $\mathcal{P}$. In fact, we obtain a functor $\Sigma: n$-Cube $(\mathcal{P}) \rightarrow \mathcal{P}$.

Suppose that $K$ is an $I$-cube and let $J_{0}, J$ be disjoint subsets of $I$ with $\# J=q$. We call the $J$-cube $\left(K_{J_{0} \cup J^{\prime}}\right)_{J^{\prime} \subset J}$ a $q$-face of $K$. If $i \in I$, we will denote by $\operatorname{Front}_{i}(K), \operatorname{Back}_{i}(K)$ the $I-\{i\}$-cubes given by $J_{0}=\{i\}, J_{0}=\emptyset$. We may think of $\operatorname{Front}_{i}(K), \operatorname{Back}_{i}(K)$ as the two "opposite" $n$-1-faces of $K$ obtained by restricting the $i$-th coordinate to be equal to 1 , resp. 0 . There is a canonical isomorphism

$$
\Sigma\left(\operatorname{Front}_{i}(K)\right)+\left(-\Sigma\left(\operatorname{Back}_{i}(K)\right)\right) \stackrel{\sim}{\longrightarrow} \Sigma(K) .
$$

Conversely, if $I=J \cup\{i\}$ and $A, B$ are two $J$-cubes, we will denote by $A \stackrel{i}{-} B$ the $I$-cube whose $i$-th back face and $i$-th front face are respectively $A$ and $B$. We obtain a canonical 
isomorphism

$$
\Sigma(B)+(-\Sigma(A)) \stackrel{\sim}{\longrightarrow} \Sigma(A \stackrel{i}{\longrightarrow} B) .
$$

Definition 4.1. By definition, a decorated $n$-cube in $\mathcal{P}$ is an $n$-cube $K$ together with isomorphisms ("2-face trivializations")

$$
m_{F}: \underline{O} \stackrel{\sim}{\longrightarrow} \Sigma(F),
$$

for each 2-face $F$ of $K$, which satisfy the following condition: Suppose that $L$ is a 3 -face of $K$ which corresponds to the subsets $J_{0}$ and $J$ as above. For each pair of indices $i, j \in J$ the 2 -face trivializations for $\operatorname{Front}_{i}(L), \operatorname{Back}_{i}(L), \operatorname{Front}_{j}(L), \operatorname{Back}_{j}(L)$ should be compatible with the canonical isomorphism

$$
\Sigma\left(\operatorname{Front}_{i}(L)\right)+\left(-\Sigma\left(\operatorname{Back}_{i}(L)\right)\right) \stackrel{\sim}{\longrightarrow} \Sigma(L) \stackrel{\sim}{\longrightarrow} \Sigma\left(\operatorname{Front}_{j}(L)\right)+\left(-\Sigma\left(\operatorname{Back}_{j}(L)\right)\right)
$$

given by (4.3).

4.a. Now let $\mathfrak{S}$ be a site and suppose that $a: \mathcal{P} \rightarrow \mathfrak{S}$ is a s.c. Picard $\mathfrak{S}$-stack. For our applications, $\mathfrak{S}=S_{\text {fppf }}$. A (decorated) $n$-cube $K$ in $\mathcal{P}$ is a (decorated) $n$-cube in the fiber category $\mathcal{P}_{T}$ for some $T \in \mathrm{Ob}(\mathfrak{S})$; in particular, all the objects $K_{v}, v \in C(I)$, satisfy $a\left(K_{v}\right)=T$.

The $n$-cubes in $\mathcal{P}$ form naturally an $\mathfrak{S}$-stack $n$-Cube $(\mathcal{P})$. There is also an $\mathfrak{S}$-stack $n$-Cube ${ }_{d}(\mathcal{P})$ whose objects are decorated $n$-cubes in $\mathcal{P}$, and morphisms are given by morphisms $\Phi=(\varphi, \phi)$ : $K \rightarrow K^{\prime}$ in $n$-Cube $(\mathcal{P})$ which are compatible with the isomorphisms (4.5) in the following sense: If $F^{\prime} \subset K^{\prime}$ is a 2-face, then let $F$ be the corresponding (via $\varphi$ ) 2-face of $K$. Let $a(\Phi)=\tau: T \rightarrow T^{\prime}$. We ask that the following diagram commutes:

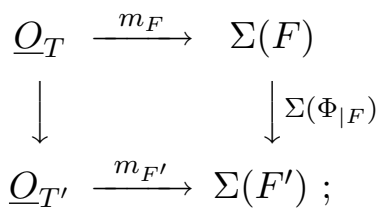

here the left vertical arrow is the canonical morphism lifting $\tau: T \rightarrow T^{\prime}$ and we denote by $\Phi_{\mid F}: F \rightarrow F^{\prime}$ the isomorphism of 2-cubes obtained from $\Phi$ "by restriction".

Now suppose that $K, K^{\prime}$ are two $I$-cubes in $\mathcal{P}$ and let $u:$ Front $_{i}(K) \stackrel{\sim}{\longrightarrow} \operatorname{Back}_{i}\left(K^{\prime}\right)$ be an isomorphism of $I-\{i\}$-cubes for some $i \in I$. Then we can define the "glueing" $K *_{i} K^{\prime}$ of $K$ and $K^{\prime}$ along $u$; this is an $I$-cube with $\operatorname{Back}_{i}\left(K *_{i} K^{\prime}\right)=\operatorname{Back}_{i}(K), \operatorname{Front}_{i}\left(K *_{i} K^{\prime}\right)=\operatorname{Front}_{i}\left(K^{\prime}\right)$. By combining the isomorphisms given by $(4.3)$ with $\Sigma(u)$ we obtain a natural isomorphism

$$
\Sigma\left(K *_{i} K^{\prime}\right) \stackrel{\sim}{\longrightarrow} \Sigma(K)+\Sigma\left(K^{\prime}\right) .
$$

If both $K$ and $K^{\prime}$ are decorated and the isomorphism $u$ is compatible with the 2-face trivializations in the sense above (i.e if it is an isomorphism of decorated cubes), then $K *_{i} K^{\prime}$ becomes naturally a decorated $I$-cube with 2-face trivializations induced by those of $K$ and $K^{\prime}([\mathrm{Du}] \S 1.5)$. 
4.b. Let $\mathcal{P}, \mathcal{Q}$ be two s.c. Picard $\mathfrak{S}$-stacks and suppose that $\delta: \mathcal{P} \rightarrow \mathcal{Q}$ is an $\mathfrak{S}$-functor. We will also denote by $\delta$ the $\mathfrak{S}$-functor

$$
\delta: n-C u b e(\mathcal{P}) \rightarrow n-C u b e(\mathcal{Q})
$$

which sends the $I$-cube $K=\left(K_{v}\right)_{v \in C(I)}$ to $\left(\delta\left(K_{v}\right)\right)_{v \in C(I)}$.

Compose with $\Sigma: n$-Cube $(\mathcal{Q}) \rightarrow \mathcal{Q}$ to obtain a functor:

$$
\delta^{n}:=\Sigma \cdot \delta: n-C u b e(\mathcal{P}) \rightarrow \mathcal{Q} ; \quad \delta^{n}(K)=\sum_{v \in C(I)}(-1)^{s(v)} \delta\left(K_{v}\right) .
$$

We can observe that if $K, K^{\prime}$ are $I$-cubes with an isomorphism $u: \operatorname{Front}_{i}(K) \stackrel{\sim}{\longrightarrow} \operatorname{Back}_{i}\left(K^{\prime}\right)$, $i \in I$, then by using (4.8) we can obtain a (natural) isomorphism

$$
\delta^{n}\left(K *_{i} K^{\prime}\right) \stackrel{\sim}{\longrightarrow} \delta^{n}(K)+\delta^{n}\left(K^{\prime}\right) .
$$

We can now restrict $\delta^{n}$ to decorated $n$-cubes to obtain a functor

$$
\delta^{n}: n-C u b e_{d}(\mathcal{P}) \rightarrow \mathcal{Q} .
$$

Let us also consider the trivial functor

$$
e: n-C u b e_{d}(\mathcal{P}) \rightarrow \mathcal{Q} ; \quad e(K)=\underline{O}, e(\Phi)=\operatorname{Id}_{\underline{O}} .
$$

Definition 4.2. ([Du] 1.6.1) An $n$-cubic structure on $\delta$ is an $\mathfrak{S}$-functor isomorphism

$$
\Xi: e \stackrel{\sim}{\longrightarrow} \delta^{n}
$$

(between functors on decorated $n$-cubes) which satisfies the following "glueing" condition: If $K, K^{\prime}$ are decorated $I$-cubes, $\# I=n$, with a (compatible) isomorphism $u: \operatorname{Front}_{i}(K) \stackrel{\sim}{\longrightarrow}$ $\operatorname{Back}_{i}\left(K^{\prime}\right)$, for some $i \in I$, then the diagram

is commutative.

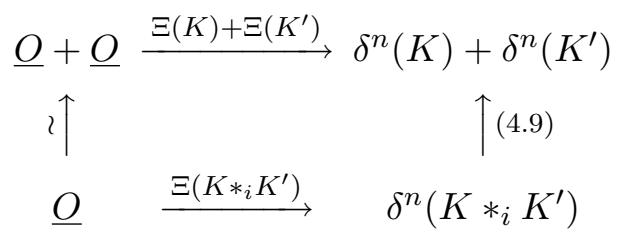

Remark 4.3. a) In [Du] 1.6.1, one actually finds a definition of the notion of $n$-cubic structure on a functor $\delta: \mathcal{P} \rightarrow \mathcal{Q}$ where $\mathcal{Q}$ is a Picard stack which is not necessarily strictly commutative. This definition involves a functor $\Sigma: n$-Cube $(\mathcal{Q}) \rightarrow \mathcal{Q}([\mathrm{Du}] 1.3 .2)$ which generalizes the functor $\Sigma$ given in the strictly commutative case above. Definition [Du] 1.6.1 requires an additional "normalization" condition on $\Xi$ which is automatically satisfied in the strictly commutative case.

b) Suppose that $K$ is a decorated $I$-cube for $I=\{1, \ldots, n\}$ and let $\sigma \in S_{n}$. The permutation $\sigma$ induces a morphism of decorated $n$-cubes $\sigma: \sigma^{*} K \rightarrow K$. This provides us with an isomorphism

$$
\delta^{n}(\sigma): \delta^{n}\left(\sigma^{*} K\right) \stackrel{\sim}{\longrightarrow} \delta^{n}(K) .
$$

Since $\Xi$ is supposed to be a functor isomorphism we must have

$$
\delta^{n}(\sigma) \cdot \Xi\left(\sigma^{*} K\right)=\Xi(K) .
$$


4.c. It follows directly from the definitions that an additive $\mathfrak{S}$-functor $F: \mathcal{P} \rightarrow \mathcal{Q}$ induces an $\mathfrak{S}$-functor between decorated $n$-cubes

$$
F: n-C u b e_{d}(\mathcal{P}) \rightarrow n-C u b e_{d}(\mathcal{Q}) .
$$

Lemma 4.4. If $\delta: \mathcal{P} \rightarrow \mathcal{Q}$ supports an n-cubic structure and $F: \mathcal{P}^{\prime} \rightarrow \mathcal{P}$ is an additive functor, then the composite $\delta \cdot F: \mathcal{P}^{\prime} \rightarrow \mathcal{Q}$ also supports an n-cubic structure.

Proof. Since by definition $(\delta \cdot F)^{n}=\delta^{n} \cdot F$, a trivialization $\Xi$ of $\delta^{n}$ defines a trivialization of $(\delta \cdot F)^{n}$. Now if $K, K^{\prime}$ are decorated $I$-cubes with a (compatible) isomorphism $u$ : Front $_{i}(K) \stackrel{\sim}{\longrightarrow} \operatorname{Back}_{i}\left(K^{\prime}\right)$, then $F(u)$ gives a compatible isomorphism between the $i$-th front and $i$-th back faces of $F(K)$ and $F\left(K^{\prime}\right)$ respectively and we have

$$
F(K) *_{i} F\left(K^{\prime}\right)=F\left(K *_{i} K^{\prime}\right)
$$

(as decorated $I$-cubes). We can now see using (4.13) that the above trivialization satisfies the glueing property of the definition.

4.d. Let $H \rightarrow S$ be a fppf abelian group scheme. By Yoneda equivalence, there is natural

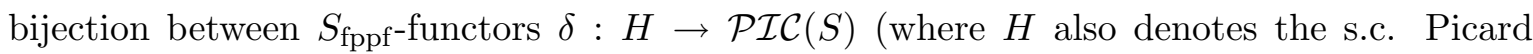
stack represented by the group scheme) and invertible $\mathcal{O}_{H}$-sheaves on the scheme $H$ given by $\delta \mapsto \mathcal{L}_{\delta}:=\delta(H \stackrel{\text { id }}{\longrightarrow} H)$.

Lemma 4.5. Suppose that the functor $\delta: H \rightarrow \mathcal{P I C}(S)$ supports an n-cubic structure in the sense of Definition 4.2 above. Then the invertible sheaf $\mathcal{L}_{\delta}$ over $H$ is equipped with an $n$-cubic structure in the sense of Definition 3.1.

Proof. For simplicity, set $\mathcal{L}=\mathcal{L}_{\delta}$. Let $I=\{1, \ldots, n\}$. We can see that, since the Picard stack $H$ is discrete, decorated $I$-cubes in $H$ correspond bijectively to ordered $n+1$-tuples $\left(a_{i}\right)_{i}, i=0, \ldots, n$, of $S^{\prime}$-valued points $a_{i} \in H\left(S^{\prime}\right)$ :

$$
\left(a_{i}\right)_{i} \mapsto K_{a_{0}}\left(a_{1}, \ldots, a_{n}\right):=\text { the cube given by } K_{v}=a_{0}+\sum_{i, v_{i}=1} a_{i} \text {. }
$$

For simplicity, if $a_{0}=0$, we will denote the above cube by $K\left(a_{1}, \ldots, a_{n}\right)$. Now notice that, by the definition of $\delta^{n}$, there is a canonical isomorphism

$$
\delta^{n}\left(K\left(a_{1}, \ldots, a_{n}\right)\right) \simeq\left(a_{1}, \ldots, a_{n}\right)^{*} \Theta_{n}(\mathcal{L}) .
$$

First suppose that $\delta$ supports an $n$-cubic structure $\Xi$; then by (4.15), the functor isomorphism $\Xi$ evaluated at the $n$-cube $K\left(a_{1}, \ldots, a_{n}\right)$ with $S^{\prime}=H^{n}$ and $a_{i}=\operatorname{pr}_{i}: H^{n} \rightarrow H$, gives a trivialization $\xi$ of the invertible sheaf $\Theta_{n}(\mathcal{L})$ over $H^{n}$. Now take all $a_{i}=0$ (as $S$-points) and set for simplicity $K_{0}=K(0, \ldots, 0)$. The $n$-cubic structure $\Xi$ provides us with an isomorphism

$$
\Xi\left(K_{0}\right): \mathcal{O}_{S} \stackrel{\sim}{\longrightarrow} \delta^{n}\left(K_{0}\right) .
$$

Now observe that $K_{0} *_{i} K_{0}=K_{0}$ for all $i$. Hence, by Definition 4.2 we obtain a commutative diagram

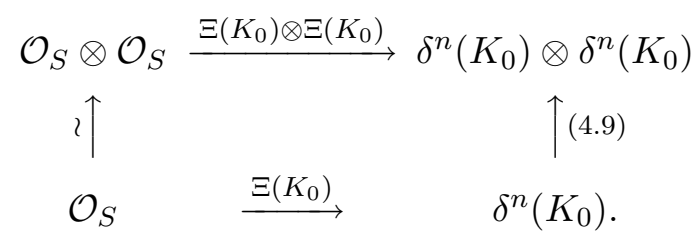


Since the contraction isomorphisms also provide us with an isomorphism $\psi_{0}: \mathcal{O}_{S} \stackrel{\sim}{\longrightarrow} \delta^{n}\left(K_{0}\right)$ that satisfies a similar commutative diagram, it follows that $\Xi\left(K_{0}\right)$ coincides with $\psi_{0}$. In view of (4.15) this implies that $\xi$ satisfies Property (c0) of Definition 3.1. To examine Property (c1) take again $S^{\prime}=H^{n}$ and $a_{i}=\operatorname{pr}_{i}: H^{n} \rightarrow H$. We have $\sigma^{*} K\left(a_{1}, \ldots, a_{n}\right)=K\left(a_{\sigma(1)}, \ldots, a_{\sigma(n)}\right)$ and under (4.15) the isomorphism (4.10) corresponds to $\mathfrak{P}_{\sigma}$ (see (3.a)). Hence, we can see that Remark 4.3 (b) implies that $\xi$ satisfies (c1) of Definition 3.1. It remains to discuss Property (c2). For this, we take $S^{\prime}=H^{n+1}$ and $a_{i}=\operatorname{pr}_{i}: H^{n+1} \rightarrow H, 0 \leq i \leq n$. Notice that we have

$$
\begin{aligned}
& K\left(a_{0}, a_{1}, a_{3}, \ldots, a_{n}\right) *_{2} K_{a_{1}}\left(a_{0}, a_{2}, \ldots, a_{n}\right)=K\left(a_{0}, a_{1}+a_{2}, a_{3}, \ldots, a_{n}\right), \\
& K\left(a_{1}, a_{2}, \ldots, a_{n}\right) *_{1} K_{a_{1}}\left(a_{0}, a_{2}, \ldots, a_{n}\right)=K\left(a_{0}+a_{1}, a_{2}, \ldots, a_{n}\right) .
\end{aligned}
$$

For example, when $n=2$ these are explained by the following diagram of three "glued" squares

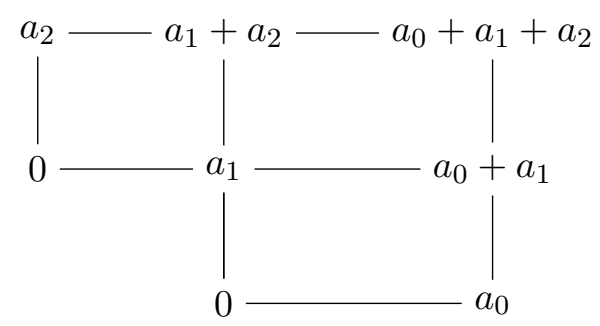

(the general case is just harder to draw: Here the vertical glueing on the right corresponds to (4.16) while the horizontal to (4.17)). The relations (4.16), (4.17) together with (4.9) give isomorphisms

$$
\begin{array}{r}
\delta\left(( K ( a _ { 0 } , a _ { 1 } , a _ { 3 } , \ldots , a _ { n } ) ) \otimes \delta \left(( K _ { a _ { 1 } } ( a _ { 0 } , a _ { 2 } , \ldots , a _ { n } ) ) \simeq \delta \left(\left(K\left(a_{0}, a_{1}+a_{2}, a_{3}, \ldots, a_{n}\right)\right),\right.\right.\right. \\
\delta\left(( K ( a _ { 1 } , a _ { 2 } , \ldots , a _ { n } ) ) \otimes \delta \left(( K _ { a _ { 1 } } ( a _ { 0 } , a _ { 2 } , \ldots , a _ { n } ) ) \simeq \delta \left(\left(K\left(a_{0}+a_{1}, a_{2}, \ldots, a_{n}\right)\right) .\right.\right.\right.
\end{array}
$$

Combining these gives:

$$
\begin{aligned}
\delta^{n}\left(K\left(a_{0}+a_{1}, a_{2}, \ldots, a_{n}\right)\right) \otimes \delta^{n} & \left.K\left(a_{0}, a_{1}, a_{3}, \ldots, a_{n}\right)\right) \simeq \\
& \simeq \delta^{n}\left(K\left(a_{0}, a_{1}+a_{2}, a_{3}, \ldots, a_{n}\right)\right) \otimes \delta^{n}\left(K\left(a_{1}, a_{2}, \ldots, a_{n}\right)\right) .
\end{aligned}
$$

We can see, by using (4.15), that this isomorphism corresponds to $\mathfrak{Q}$ of (3.a). It now follows that the glueing condition on $\Xi$ implies that the trivialization $\xi$ respects the isomorphism $\mathfrak{Q}$. In other words, Property (c2) of Definition 3.1 is true for $\xi$.

4.e. Let $h: Y \rightarrow S$ be a projective and flat morphism of relative dimension $d$ over the locally Noetherian scheme $S$. If $\mathcal{H}$ is a locally free coherent sheaf of $\mathcal{O}_{Y}$-modules on $Y$ then the total derived image $\mathbf{R} h_{*}(\mathcal{H})$ in the derived category $D^{+}\left(\mathcal{O}_{S}\right)$ of the homotopy category of complexes of sheaves of $\mathcal{O}_{S}$-modules which are bounded below is "perfect" (i.e it is locally on $S$ quasi-isomorphic to a bounded complex of finitely generated free $\mathcal{O}_{S}$-modules, see [SGA6] III). Hence, by $[\mathrm{KM}]$, we can associate to $\mathbf{R} h_{*}(\mathcal{H})$ a graded invertible sheaf

$$
\operatorname{det}_{*} \mathbf{R} h_{*}(\mathcal{H})=\left(\operatorname{det} \mathbf{R} h_{*}(\mathcal{H}), \operatorname{rk}\left(\mathbf{R} h_{*}(\mathcal{H})\right)\right.
$$


on $S$ (the "determinant of cohomology"). By restricting to $\mathcal{H}$ which are invertible, we obtain a functor, which we will denote again by $\operatorname{det}_{*} \mathbf{R} h_{*}$, from the Picard category of invertible sheaves on $Y$ to the Picard category of graded invertible sheaves on $S$. In fact, by loc. cit. the formation of the determinant of cohomology commutes with arbitrary base changes $S^{\prime} \rightarrow S$; hence we can define a corresponding $S_{\text {fppf }}$-functor

$$
\operatorname{det}_{*} \mathbf{R} h_{*}: \mathcal{P} \mathcal{I C}(Y) \rightarrow \mathcal{P} \mathcal{I C}{ }_{*}(S) .
$$

By the main result of $[\mathrm{Du}]$ (Theorem 4.2) this functor supports a canonical $d+2$-cubic structure (in the sense of [Du] Definition 1.6.1). Let us remark here that since $\mathcal{P I C}_{*}(S)$ is not strictly commutative we do have to refer to $[\mathrm{Du}]$ for the definition of cubic structure. (See Remark 4.3 (a).) Now observe that the substack $\mathcal{P} \mathcal{I C}$ ev $(S)$ of $\mathcal{P} \mathcal{I C} \mathcal{C}_{*}(S)$ given by graded line bundles $(\mathcal{M}, e)$ with $e$ always even is a s.c. Picard stack. The forgetful functor $f$ : $\mathcal{P I C}_{\text {ev }}(S) \rightarrow \mathcal{P I C}(S)$ is an additive functor. (Note that this is not true for the forgetful functor $\mathcal{P I C}_{*}(S) \rightarrow \mathcal{P} \mathcal{I C}(S)$.) This fact together with [Du] Theorem 4.2 and Remark 4.3 (a) implies the following:

i) The functor $\mathcal{P} \mathcal{I C}(Y) \rightarrow \mathcal{P I C}(S)$ given by $\mathcal{H} \mapsto \operatorname{det} \mathbf{R} h_{*}(\mathcal{H})^{\otimes 2}$ supports a canonical $d+2$-cubic structure in the sense of Definition 4.2.

ii) Let $\mathcal{P} \mathcal{I} \mathcal{C}^{\mathrm{ev}}(Y)$ be the substack of $\mathcal{P} \mathcal{I C}(Y)$ given by invertible sheaves for which the function $\operatorname{rk}\left(\mathbf{R} h_{*}(\mathcal{H})\right)$ is always even. Then the functor $\mathcal{P} \mathcal{I} \mathcal{C}^{\mathrm{ev}}(Y) \rightarrow \mathcal{P} \mathcal{I C}(S)$ given by $\mathcal{H} \mapsto$ $\operatorname{det} \mathbf{R} h_{*}(\mathcal{H})$ supports a canonical $d+2$-cubic structure in the sense of Definition 4.2.

Remark 4.6. In fact, one can give a somewhat more direct proof of (i) and (ii) by following the general strategy of the proof of the main theorem of $[\mathrm{Du}]$. The argument is considerably less involved since we do not have to deal with the thorny problem of signs that complicates the proof of $[\mathrm{Du}]$ Theorem 4.2 .

4.f. Let $h: Y \rightarrow S$ be as in the previous paragraph and assume in addition that $S$ is the spectrum of a Dedekind ring $R$ with field of fractions $K$. Suppose that $\pi: X \rightarrow Y$ is a $G$ torsor with $G$ a finite abelian group. The construction of $\S 2$.e gives an additive $S_{\text {fppf }}$-functor

$$
F_{\pi}: G_{S}^{D} \rightarrow \mathcal{P} \mathcal{I C}(Y) ; \quad F(\chi)=\mathcal{O}_{X, \chi} .
$$

Let $h_{G^{D}}: Y \times_{S} G_{S}^{D} \rightarrow G_{S}^{D}$ be the base change of $h: Y \rightarrow S$; we can view $\pi_{*}\left(\mathcal{O}_{X}\right)$ as an invertible sheaf on $Y \times{ }_{S} G^{D}$ which is isomorphic to the value $\mathcal{O}_{X, \chi_{0}}$ of the functor $F$ on the "universal" point $\chi_{0}$ (see (2.2)). It now follows from (2.2) and (2.3) that the invertible

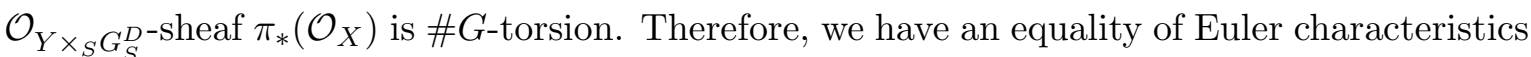
(ranks)

$$
\operatorname{rk}\left(\mathbf{R} h_{G^{D}}\left(\pi_{*}\left(\mathcal{O}_{X}\right)\right)\right)=\operatorname{rk}\left(\mathbf{R} h_{G^{D} *}\left(\mathcal{O}_{Y \times_{S} G_{S}^{D}}\right)\right) .
$$

(This can be deduced from the Grothendieck-Riemann-Roch theorem or more simply as follows: In the Grothendieck group $\mathrm{K}_{0}\left(Y \times{ }_{S} G_{S}^{D}\right)$ the rank zero element $z=\left[\pi_{*}\left(\mathcal{O}_{X}\right)\right]-\left[\mathcal{O}_{Y \times{ }_{S} G^{D}}\right]$ is nilpotent. Now since $\pi_{*}\left(\mathcal{O}_{X}\right)$ is a \#G-power torsion invertible sheaf, it follows that $z$ is torsion. Hence, $z$ has Euler characteristic equal to zero and this implies the equality.) Using the projection formula and the flatness of $Y \rightarrow S$, we see that the locally constant function $G_{S}^{D} \rightarrow \mathbf{Z}$ given by $\operatorname{rk}\left(\mathbf{R} h_{G^{D} *}\left(\mathcal{O}_{Y \times_{S} G_{S}^{D}}\right)\right)$ always takes the value $\chi\left(Y, \mathcal{O}_{Y}\right)=\chi\left(Y_{K}, \mathcal{O}_{Y_{K}}\right):=$ $\sum_{i}(-1)^{i} \mathrm{rk}_{K} \mathrm{H}^{i}\left(Y_{K}, \mathcal{O}_{Y_{K}}\right)$. We can now conclude using base change that for any $S^{\prime}$-valued 
point $\chi$ of $G_{S}^{D}$ the function $\operatorname{rk}\left(\mathbf{R} h_{S^{\prime} *}\left(\mathcal{O}_{X, \chi}\right)\right)$ is constant with value $\chi\left(Y_{K}, \mathcal{O}_{Y_{K}}\right)$. This value is even if and only if the arithmetic genus $g\left(Y_{K}\right)=(-1)^{d}\left(\chi\left(Y_{K}, \mathcal{O}_{Y_{K}}\right)-1\right)$ of the generic fiber is odd. Now let $\kappa=\operatorname{gcd}\left(2, g\left(Y_{K}\right)\right)$ and consider the composition

$$
\delta:=\operatorname{det} \mathbf{R} h_{*}^{\otimes \kappa} \cdot F_{\pi}: G_{S}^{D} \rightarrow \mathcal{P} \mathcal{I C}(S)
$$

given by $\chi \mapsto \operatorname{det} \mathbf{R} h_{*}\left(\mathcal{O}_{X, \chi}\right)^{\otimes \kappa}$. By Lemma 4.4 and (i), (ii) of $\S 4$.e above (applied when $g\left(Y_{K}\right)$ is even, odd respectively) we conclude that the functor $\delta$ has a canonical $d+2$-cubic structure in the sense of Definition 4.2. Using Lemma 4.5 and $\pi_{*}\left(\mathcal{O}_{X}\right)=\mathcal{O}_{X, \chi_{0}}$, we see that the $d+2$-cubic structure on $\delta$ equips the invertible sheaf $\mathcal{L}_{\delta}=\operatorname{det} \mathbf{R} h_{G^{D}}\left(\pi_{*}\left(\mathcal{O}_{X}\right)\right)^{\otimes \kappa}$ over $G_{S}^{D}$ with a corresponding $d+2$-cubic structure in the sense of Definition 3.1. We summarize:

Theorem 4.7. Let $h: Y \rightarrow S$ be a projective and flat morphism of relative dimension d over the spectrum of a Dedekind ring with field of fractions $K$; set $\kappa=\operatorname{gcd}\left(2, g\left(Y_{K}\right)\right)$ with $g\left(Y_{K}\right)=(-1)^{d}\left(\chi\left(Y_{K}, \mathcal{O}_{Y_{K}}\right)-1\right)$ the arithmetic genus of the generic fiber of $Y \rightarrow S$. Suppose that $\pi: X \rightarrow Y$ is a $G$-torsor for the finite abelian group $G$. Then the invertible sheaf $\operatorname{det} \mathbf{R} h_{G^{D}}\left(\pi_{*}\left(\mathcal{O}_{X}\right)\right)^{\otimes \kappa}$ over $G_{S}^{D}$ supports a $d+2$-cubic structure.

\section{Galois module structure}

In this section, we prove the results stated in the introduction. We start by recalling the definition of the projective Euler characteristic.

5.a. Suppose that $S=\operatorname{Spec}(R), R$ a Dedekind ring, and that $\pi: X \rightarrow Y$ is a (right) torsor for a finite group $G$ with $h: Y \rightarrow S$ projective. Set $f=\pi \cdot h: X \rightarrow S$. Denote by $\mathrm{G}_{0}(G, X)$ the Grothendieck group of $G$-equivariant coherent sheaves on $X$. By descent (see $\S 2 . \mathrm{d})$ pulling back along $\pi$ gives an isomorphism $\pi^{*}: \mathrm{G}_{0}(Y) \stackrel{\sim}{\longrightarrow} \mathrm{G}_{0}(G, X)$. Recall that if $\mathcal{F}$ is a $G$-equivariant coherent sheaf on $X$ we can view $\pi_{*}(\mathcal{F})$ as a coherent sheaf of $\mathcal{O}_{Y}[G]$ modules on $Y$ (cf. $\S 2$.d). We can then consider the right derived image $\mathbf{R} \Gamma\left(Y, \pi_{*}(\mathcal{F})\right)$; this is a complex in $D^{+}(R[G])$ which computes the cohomology of $\pi_{*}(\mathcal{F})$ ([SGA6] III $\S 2$, IV $\S 2$ ). We may give a complex isomorphic to $\mathbf{R} \Gamma\left(Y, \pi_{*}(\mathcal{F})\right)$ by taking the (bounded) Cech complex obtained by considering the sections of $\pi_{*}(\mathcal{F})$ on the intersections of the sets in a finite affine cover $\left\{V_{i}\right\}_{i}$ of $Y$.

Theorem 5.1. ([CEPT1] Theorem 8.3; see also [C], [CE].) Assume that $\pi: X \rightarrow Y$ is a G-torsor and $h: Y \rightarrow S=\operatorname{Spec}(R)$ is projective with $R$ a Dedekind ring. Let $\mathcal{F}$ be a $G$-equivariant coherent sheaf on $X$. Then the complex $\mathbf{R} \Gamma\left(Y, \pi_{*}(\mathcal{F})\right)$ in $D^{+}(R[G])$ is perfect. The elements

$$
\chi_{f}^{P}(\mathcal{F}):=\chi\left(\mathbf{R} \Gamma\left(Y, \pi_{*}(\mathcal{F})\right)\right) \in \mathrm{K}_{0}(R[G])
$$

define a group homomorphism (the equivariant projective Euler characteristic)

$$
\chi_{f}^{P}: \mathrm{G}_{0}(G, X) \rightarrow \mathrm{K}_{0}(R[G]) .
$$

We also set $\bar{\chi}_{f}^{P}(\mathcal{F}):=\left(\chi_{f}^{P}(\mathcal{F})\right)^{\text {red }} \in \mathrm{K}_{0}^{\mathrm{red}}(R[G])$. Note that $\bar{\chi}_{f}^{P}(\mathcal{F}) \in \mathrm{K}_{0}^{\mathrm{red}}(R[G])$ is the obstruction for the complex $\mathbf{R} \Gamma\left(Y, \pi_{*}(\mathcal{F})\right)$ to be isomorphic in $D^{+}(R[G])$ to a bounded complex of finitely generated free $R[G]$-modules. When $f: X \rightarrow S$ is fixed, we will usually write $\chi^{P}$, 
$\bar{\chi}^{P}$ instead of $\chi_{f}^{P}, \bar{\chi}_{f}^{P}$. Let us note here that if $R$ is the ring of integers of a number field then that $\mathrm{K}_{0}^{\mathrm{red}}(R[G])$ coincides with the "class group" $\mathrm{Cl}(R[G])$ of $[\mathrm{F}]$.

5.b. We now assume, in addition, that $G$ is commutative and that the morphism $h$ is flat.

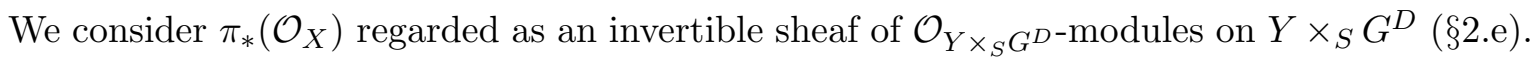
As in $\S 4$.f, the complex $\mathbf{R} h_{G^{D}}\left(\pi_{*}\left(\mathcal{O}_{X}\right)\right)$ in the derived category $D^{+}\left(\mathcal{O}_{G^{D}}\right)$ is perfect and we can consider the determinant of cohomology $\operatorname{det} \mathbf{R} h_{G^{D}}(\mathcal{H})$ and the Euler characteristic $\chi\left(\mathbf{R} h_{G^{D}}(\mathcal{H})\right) \in \mathrm{K}_{0}(R[G])$. (Notice that, by $[\mathrm{KM}]$ Prop. 4, over the affine scheme $G^{D}$, a complex is perfect in the sense of [SGA6] if and only if it is globally quasi-isomorphic to a bounded complex of sheaves associated to finitely generated locally free $R[G]$-modules; this allows us to define $\chi\left(\mathbf{R} h_{G^{D}}(\mathcal{H})\right) \in \mathrm{K}_{0}(R[G])$ by a formula similar to (2.1).) We can construct a bounded complex isomorphic to $\mathbf{R} h_{G^{D}}\left(\pi_{*}\left(\mathcal{O}_{X}\right)\right)$ by using the Cech construction associated to $\pi_{*}\left(\mathcal{O}_{X}\right)$ and the finite affine cover $\left\{V_{i} \times{ }_{S} G^{D}\right\}_{i}$ of $Y \times_{S} G^{D}$, where $\left\{V_{i}\right\}_{i}$ is a finite affine cover of $Y$. It follows that the complexes $\mathbf{R} h_{G^{D}}\left(\pi_{*}\left(\mathcal{O}_{X}\right)\right)$ and $\mathbf{R} \Gamma\left(Y, \pi_{*}\left(\mathcal{O}_{X}\right)\right)$ are isomorphic (here again we identify $R[G]$-modules with the corresponding $\mathcal{O}_{G^{D}}$-sheaves). We obtain

$$
i\left(\bar{\chi}_{f}^{P}\left(\mathcal{O}_{X}\right)\right):=i\left(\chi\left(\mathbf{R} \Gamma\left(Y, \pi_{*}\left(\mathcal{O}_{X}\right)\right)\right)^{\mathrm{red}}\right)=\left[\operatorname{det} \mathbf{R} h_{G^{D}}\left(\pi_{*}\left(\mathcal{O}_{X}\right)\right)\right]
$$

in $\operatorname{Pic}\left(G_{S}^{D}\right)=\operatorname{Pic}(R[G])$. After identifying $\mathrm{K}_{0}^{\operatorname{red}}(R[G])$ with $\operatorname{Pic}(R[G])=\operatorname{Pic}\left(G_{S}^{D}\right)$ we have

$$
\bar{\chi}_{f}^{P}\left(\mathcal{O}_{X}\right)=\left[\operatorname{det} \mathbf{R} h_{G^{D}}\left(\pi_{*}\left(\mathcal{O}_{X}\right)\right)\right] .
$$

5.c. We continue with the general assumptions of the previous paragraph. In particular, $G$ is commutative and $\pi: X \rightarrow Y$ is a $G$-torsor with $h: Y \rightarrow S$ projective and flat. By Remark 2.1 and (2.2) the $\mathbf{G}_{m}$-torsor that corresponds to the invertible sheaf $\pi_{*}\left(\mathcal{O}_{X}\right)$ over $G_{Y}^{D}=Y \times_{S} G_{S}^{D}$ supports the structure of a commutative extension

$$
1 \rightarrow \mathbf{G}_{m Y} \rightarrow E \rightarrow G_{Y}^{D} \rightarrow 1
$$

of group schemes over $Y$.

Definition 5.2. We will say that the $G$-torsor $\pi: X \rightarrow Y$ is of Albanese type over $S$ if there is a smooth commutative group scheme of finite type $A \rightarrow S$ with connected fibers, a commutative extension

$$
1 \rightarrow \mathbf{G}_{m Y} \rightarrow \mathcal{E} \rightarrow A_{Y} \rightarrow 1
$$

of group schemes over $Y$ and a group scheme homomorphism $\phi: G_{S}^{D} \rightarrow A$ over $S$ such that $E \simeq\left(\operatorname{id}_{Y} \times_{S} \phi\right)^{*}(\mathcal{E})$ as group scheme extensions.

Remark 5.3. a) If $\mathcal{P}$ is the invertible sheaf over $A_{Y}=Y \times_{S} A$ which corresponds to the $\mathbf{G}_{m Y}$-torsor $\mathcal{E}$, then our condition implies that $\pi_{*}\left(\mathcal{O}_{X}\right) \simeq\left(\operatorname{id}_{Y} \times_{S} \phi\right)^{*}(\mathcal{P})$ as invertible shaves on $G_{Y}^{D}$.

b) Let $\pi: X \rightarrow Y$ be a $G$-torsor of Albanese type and suppose that $i: Z \rightarrow Y$ is a projective morphism such that $h \cdot i: Z \rightarrow S$ is flat. Then the $G$-torsor $\pi_{Z}: X \times_{Y} Z \rightarrow Z$ obtained by base change is also of Albanese type.

c) Suppose $S=\operatorname{Spec}(k)$ with $k$ a field of characteristic prime to the order of $G$ and that $Y$ is smooth and projective over $\operatorname{Spec}(k)$. Assume that $Y$ contains a $k$-rational point. The following construction motivates our terminology: Take $A=\operatorname{Pic}^{0}(Y)$ (the Picard abelian 
variety of $Y$ ) and suppose we have a group scheme immersion $\phi: G^{D} \rightarrow A$. The $k$-rational point on $Y$ provides us with a morphism $Y \rightarrow \operatorname{Alb}(Y)$ to the Albanese abelian variety of $Y$. Set $B=A / G^{D}$ for the quotient abelian variety and consider

$$
0 \rightarrow G \rightarrow B^{\text {dual }} \rightarrow A^{\text {dual }} \rightarrow 0
$$

given by the isogeny dual to $A \rightarrow B$. The canonical duality $A^{\text {dual }} \simeq \operatorname{Alb}(Y)$ between the Albanese and Picard varieties of $Y$ allows us to view this exact sequence as a $G$-torsor over $\operatorname{Alb}(Y)$. We can restrict this along $Y \rightarrow \operatorname{Alb}(Y)$ to obtain a $G$-torsor $X \rightarrow Y$. Such torsors are called of Albanese type by Lang [La]. One can now see (using for example [Mu] $\S 15$ ) that $X \rightarrow Y$ is also of Albanese type according to our definition above. The required extension (5.3) is given as the pull-back along $Y \times_{S} A \rightarrow \operatorname{Alb}(Y) \times_{S} A \simeq A^{\text {dual }} \times_{S} A$ of the extension over $A_{A^{\text {dual }}}=A^{\text {dual }} \times{ }_{S} A$ given by the Poincare invertible sheaf with its biextension structure.

Theorem 5.4. Suppose $S=\operatorname{Spec}(k)$ with $k$ a field of characteristic prime to the order of $G$ and that $Y$ is smooth and projective over $\operatorname{Spec}(k)$. Assume that $Y$ contains a k-rational point and let $X \rightarrow Y$ be a G-torsor such that $X$ is geometrically connected. Then $X \rightarrow Y$ is of Albanese type if and only if all the torsion invertible sheaves $\mathcal{O}_{X, \chi}$, with $\chi$ running over all characters of $G$ (see §2.e), correspond to divisors on $Y \times_{k} k(\chi)$ which are algebraically trivial, or equivalently if and only if $X \rightarrow Y$ is obtained by pulling back along $Y \rightarrow \operatorname{Alb}(Y)$ the cover given by an isogeny with kernel $G$ of the Albanese $\operatorname{Alb}(Y)$.

Proof. This follows from the construction and properties of the Picard and Albanese abelian varieties of $Y$ as in Remark 5.3 (c) above.

Remark 5.5. Assume that $X \rightarrow Y$ is an abelian $G$-torsor over the ring of integers $\mathcal{O}_{K}$ of the number field $K$ such that the generic fiber $X_{K} \rightarrow Y_{K}$ is of Albanese type. Assume that $Y_{K}$ is smooth and that $Y \rightarrow \operatorname{Spec}\left(\mathcal{O}_{K}\right)$ has a section. Let $\mathcal{A}^{0}$ be the connected component of the Néron model $\mathcal{A}$ of $\operatorname{Pic}^{0}\left(Y_{K}\right)$. It would be interesting to find conditions under which $X \rightarrow Y$ is of Albanese type over $\operatorname{Spec}\left(\mathcal{O}_{K}\right)$ with $A=\mathcal{A}^{0}$; one can see using [Ra] $\S 8$ that this is always the case when $Y_{K}$ is a curve of genus $\geq 2$ and $Y$ is regular.

Let $\pi: X \rightarrow Y$ be a $G$-torsor of Albanese type over $S=\operatorname{Spec}(R)$. Suppose that the fraction field of $R$ is perfect. Denote by $h_{A}: Y \times_{S} A \rightarrow A$ the base change of $h$ and let $\mathcal{M}_{A}=$ $\operatorname{det}\left(\mathbf{R} h_{A *}(\mathcal{P})\right), \mathcal{M}=\operatorname{det} \mathbf{R} h_{G^{D}}\left(\pi_{*}\left(\mathcal{O}_{X}\right)\right.$ ) (invertible sheaves on $A$ and $G_{S}^{D}$ respectively). Since $\pi_{*}\left(\mathcal{O}_{X}\right) \simeq\left(\operatorname{id}_{Y} \times_{S} \phi\right)^{*}(\mathcal{P})$ we then have $\mathcal{M} \simeq \phi^{*}\left(\mathcal{M}_{A}\right)$. By [Br] Proposition 2.4 and the discussion before it (see also [SGA7I] VIII), the invertible sheaf $\mathcal{M}_{A}$ over $A$ supports a canonical cubic structure (as in Definition 3.1 with $n=3$ ). This statement is of course an extension to this situation of the classical theorem of the cube for line bundles over abelian varieties. (Notice that Breen uses a slightly different definition of cubic structure; see [P2] Remark 3.2 (a) or [Br] 2.8. However, it is not hard to see that this does not affect the truth of our statement.) Using the functoriality of cubic structures, we can conclude that $\mathcal{M} \simeq \phi^{*}\left(\mathcal{M}_{A}\right)$ also supports a cubic structure.

5.d. We can now complete the proof of our main results (Theorems 1.3, 1.4 and 1.5).

As in these statements we assume that $G$ is a finite group and $\pi: X \rightarrow Y$ is a $G$-torsor with $h: Y \rightarrow S=\operatorname{Spec}(\mathbf{Z})$ projective and flat of relative dimension $d$. We set $f=h \cdot \pi$. 
A standard argument using Noetherian induction shows:

Lemma 5.6. The Grothendieck group $\mathrm{G}_{0}(Y)$ is generated by the classes $\left[i_{*} \mathcal{O}_{Z}\right]$ with $i: Z \hookrightarrow$ $Y$ an integral subscheme of $Y$. There are two possibilities for such a $Z$ : Either the morphism $Z \rightarrow \operatorname{Spec}(\mathbf{Z})$ is flat of relative dimension $d^{\prime} \leq d$, or it factors through $\operatorname{Spec}\left(\mathbf{F}_{p}\right) \rightarrow \operatorname{Spec}(\mathbf{Z})$ for some prime $p$.

Recall the descent isomorphism $\pi^{*}: G_{0}(Y) \stackrel{\sim}{\longrightarrow} G_{0}(G, X)$. Let $i: Z \hookrightarrow Y$ be as in Lemma 5.6 and consider the $G$-torsor $\pi_{Z}: X \times_{Y} Z \rightarrow Z$ obtained by pulling back $\pi$ along the closed immersion $i$. Denote by $f^{\prime}: X \times_{Y} Z \rightarrow \operatorname{Spec}(\mathbf{Z})$ the structure morphism. Then it follows from the construction of the Euler characteristic that

$$
\chi_{f}^{P}\left(\pi^{*}\left(i_{*} \mathcal{O}_{Z}\right)\right)=\chi_{f^{\prime}}^{P}\left(\mathcal{O}_{X \times_{Y} Z}\right) .
$$

If $Z \rightarrow \operatorname{Spec}(\mathbf{Z})$ factors through $\operatorname{Spec}\left(\mathbf{F}_{p}\right) \rightarrow \operatorname{Spec}(\mathbf{Z})$ then it follows from the main theorem of [Na] that $\bar{\chi}_{f^{\prime}}^{P}\left(\mathcal{O}_{X \times_{Y} Z}\right)=0$ (see also [P1] 4.b Remark 3). On the other hand, we notice that if $d^{\prime} \leq d$ we have $M_{d^{\prime}+1}(G)\left|M_{d+1}(G), M_{d^{\prime}+1}^{\prime}(G)\right| M_{d+1}^{\prime}(G)$ and also $C_{d^{\prime}+1}(G) \mid C_{d+1}(G)$. Using Lemma 5.6, the additivity of the Euler characteristic $\bar{\chi}_{f}^{P}$ (Theorem 5.1) and (5.4), we can now see that the proofs of Theorems 1.3, 1.4 are reduced to the case that $\mathcal{F}$ is the structure sheaf $\mathcal{O}_{X}=\pi^{*} \mathcal{O}_{Y}$ and $Y$ is integral. A similar argument using $\left(d^{\prime}+1\right) ! ! \mid(d+1) !$ ! for $d^{\prime} \leq d$ together with Remark 5.3 (b) shows that the proof of Theorem 1.5 is also reduced to the case that $\mathcal{F}$ is the structure sheaf of $X$ and $Y$ is integral.

Let us first discuss the completion of the proofs of Theorems 1.3 and 1.4. Noetherian induction and an argument as in the proof of [P1] Proposition 4.4 (a) shows that $(\# G)^{d+1}$. $\bar{\chi}^{P}\left(\mathcal{O}_{X}\right)=0$. Suppose first that $G$ is abelian. In this case, by Theorem 4.7, the invertible sheaf $\operatorname{det} \mathbf{R} h_{G^{D}}\left(\pi_{*}\left(\mathcal{O}_{X}\right)\right)^{\otimes \kappa}$ over $G_{S}^{D}$ supports a $d+2$-cubic structure. Hence, it follows from (5.1) and Theorem 3.2 that $\kappa \cdot \bar{\chi}^{P}\left(\mathcal{O}_{X}\right)$ is annihilated by $M_{d+1}(G)$. Since $(\# G)^{d+1} \cdot \bar{\chi}^{P}\left(\mathcal{O}_{X}\right)=0$ this gives Theorem 1.3 (b) for $\mathcal{F}=\mathcal{O}_{X}$ when $G$ is abelian.

The fact that $(\# G)^{d+1} \cdot \bar{\chi}^{P}\left(\mathcal{O}_{X}\right)=0$ together with the "localization" argument in the proof of [P1] Proposition 4.5 shows that the proofs of Theorems 1.3 and 1.4 in general reduce to the case that $G$ is an $l$-group, $l$ prime.

Theorem 1.3 (b) now follows from the abelian case just explained above. To show Theorem 1.3 (a) observe that the argument in [P1] p. 215-216 allows us to reduce the case of an $l$-group to that of the case of a "basic" $l$-group with $l$ odd, i.e to the case of a cyclic group of odd prime order $l$. Then part (a) follows once again by the abelian case. This completes the proof of Theorem 1.3.

The same argument from [P1] p. 215-216 also shows that the proof of Theorem 1.4 can be reduced to the case of a cyclic group of odd prime order $l$. Then Theorem 1.4 follows from Theorem 4.7 and Theorem 3.3.

Let us now discuss the proof of Theorem 1.5. Recall that it is enough to deal with the case that $\mathcal{F}=\mathcal{O}_{X}$ and $Y$ is integral. It is also enough to assume that $d \geq 1$. Let us first discuss part (a): Since $\pi: X \rightarrow Y$ is of Albanese type, by $\S 5 . c$, the invertible sheaf $\mathcal{M}:=\operatorname{det} \mathbf{R} h_{G^{D}}\left(\pi_{*}\left(\mathcal{O}_{X}\right)\right)$ supports a 3 -cubic structure. Hence, by Theorem 3.2 and the comment directly below its statement, $\mathcal{M}$ is trivial. It follows from (5.1) that $\bar{\chi}^{P}\left(\mathcal{O}_{X}\right)=0$ in $\mathrm{Cl}(\mathbf{Z}[G])$. This completes the proof of part (a). 
It remains to prove Theorem $1.5(\mathrm{~b})$ :

For simplicity, we set $T=X_{\mathbf{Q}}, U=Y_{\mathbf{Q}}$. We are assuming that $T \rightarrow U$ is of Albanese type and so there is a commutative group scheme $A$ and an extension $\mathcal{E}$ as in Definition 5.2. We denote by $\mathcal{P}$ the invertible sheaf over $A_{U}=U \times_{\mathbf{Q}} A$ given by $\mathcal{E}$. By Theorem 4.7, the invertible sheaf $\mathcal{M}^{\otimes 2}=\operatorname{det} \mathbf{R} h_{G^{D}}\left(\pi_{*}\left(\mathcal{O}_{X}\right)\right)^{\otimes 2}$ over $G_{S}^{D}$ supports a $d+2$-cubic structure $\xi$. We can apply the same argument to the invertible sheaf $\mathcal{M}_{A}=\operatorname{det}\left(\mathbf{R} h_{A *}(\mathcal{P})\right)$ over $A$ : Here we start from the extension

$$
1 \rightarrow \mathbf{G}_{m U} \rightarrow \mathcal{E} \rightarrow A_{U} \rightarrow 1
$$

and the corresponding additive functor $A \rightarrow \mathcal{P I C}(U)$. We obtain that $\mathcal{M}_{A}^{\otimes 2}$ has a canonical $d+2$-cubic structure $\xi_{A}$ over $A$. Recall that the extension $\mathcal{E}$ pulls back to the extension

$$
1 \rightarrow \mathbf{G}_{m U} \rightarrow E \rightarrow G_{U}^{D} \rightarrow 1
$$

using $\phi: G_{\mathbf{Q}}^{D}=G_{\mathrm{Spec}(\mathbf{Q})}^{D} \rightarrow A$. By Remark 2.1 and the construction of the $d+2$-cubic structure $\xi_{A}$ this implies that the $d+2$-cubic structure $\xi_{\mathbf{Q}}$ on the generic fiber $\mathcal{M}_{\mathbf{Q}}^{\otimes 2}$ (an invertible sheaf over $\left.G_{\mathbf{Q}}^{D}=\operatorname{Spec}(\mathbf{Q}[G])\right)$ is obtained by pulling back $\xi_{A}$ along $\phi$; so there is an isomorphism of $d+2$-cubic structures $\xi_{\mathbf{Q}} \simeq \phi^{*}\left(\xi_{A}\right)$. However, as a corollary of the theorem of the cube on $A$ (see the argument at the end of the previous paragraph), $\mathcal{M}_{A}$ and hence also $\mathcal{M}_{A}^{\otimes 2}$ has a canonical 3-cubic structure. This induces a $d+2$-cubic structure $\xi_{A}^{\prime}$ on $\mathcal{M}_{A}^{\otimes 2}$ using the construction described in [P2] Lemma 5.1. (The result is stated in loc. cit. for cubic structures on the Cartier dual of a finite abelian group; however, the construction easily extends to our case of an abelian scheme $A$. Indeed, in this case, if a trivialization as in (3.1) satisfies (c0) then it also satisfies conditions (c1) and (c2); see also below.) We claim that this agrees with the $d+2$-cubic structure $\xi_{A}$ : To see this observe that the composition $\xi_{A}^{-1} \cdot \xi_{A}^{\prime}$ is given by an invertible regular function $c$ on $A^{d+2}$ which gives a $d+2$-cubic structure on the trivial invertible sheaf. This implies that we have $c\left(a_{1}, \ldots, a_{d+2}\right)=1$ if one of the $a_{i}$ is zero (cf. [P2] §3.b, (c0')). A classical lemma of Rosenlicht ([SGA7] VIII 4.1) now gives that $c$ is equal to 1 . Hence $\xi_{A}^{\prime}=\xi_{A}$. Since $\xi_{\mathbf{Q}} \simeq \phi^{*}\left(\xi_{A}\right)$ and $\xi_{A}^{\prime}=\xi_{A}$ comes from a 3-cubic structure (using [P2] Lemma 5.1) we conclude that $\xi_{\mathbf{Q}}$ also comes from a 3-cubic structure. Now we will consider the polynomial expansion of [P2] Corollary 5.6 for $\left(\mathcal{M}^{\otimes 2}, \xi\right)$ and $\left(\mathcal{M}_{\mathbf{Q}}^{\otimes 2}, \xi_{\mathbf{Q}}\right)$ (we take $n=d+1)$. For simplicity, set $\mathcal{L}=\mathcal{M}^{\otimes 2}, \mathcal{L}_{\mathbf{Q}}=\mathcal{M}_{\mathbf{Q}}^{\otimes 2}$. By repeated application of [P2] Lemma 5.2 and [P2] Proposition 5.4 we see that, since $\left(\mathcal{L}_{\mathbf{Q}}, \xi_{\mathbf{Q}}\right)$ is a $d+2$-cubic structure which comes from a 3-cubic structure, the $(d+1-i)$-extensions $E\left(\mathcal{L}_{\mathbf{Q}}^{(i)}, \xi_{\mathbf{Q}}^{(i)}\right)$ of $G_{\mathbf{Q}}^{D}$ by $\mathbf{G}_{m}$ are trivial when $d+1-i \geq 3$. Since the construction of $\left(\mathcal{L}^{(i)}, \xi^{(i)}\right)$ commutes with base change from $\mathbf{Z}$ to $\mathbf{Q}$, this now implies that the $(d+1-i)$-extensions $E\left(\mathcal{L}^{(i)}, \xi^{(i)}\right)$ of $G^{D}$ by $\mathbf{G}_{m}$ (over $S=\operatorname{Spec}(\mathbf{Z})$ ) become trivial after base change to the generic fiber $\operatorname{Spec}(\mathbf{Q})$ when $d+1-i \geq 3$. We claim that this implies that all these multiextensions are trivial. To see this let us consider the commutative diagram

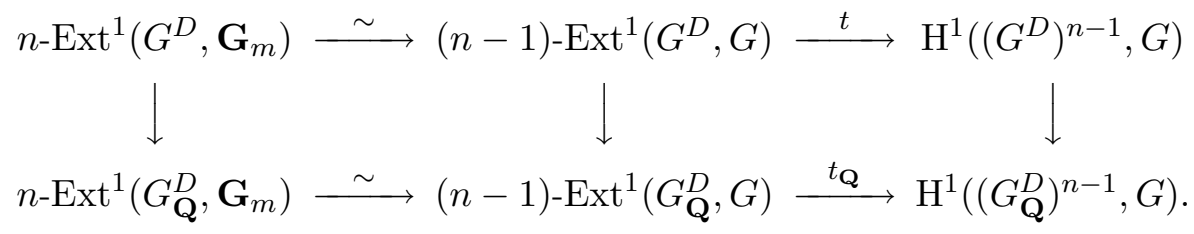


Here the horizontal isomorphisms on the left side are given by [P2] (6.12). The homomorphism $t$ is the forgetful map; the bottom row is obtained by the constructions that give the first row but performed over $\operatorname{Spec}(\mathbf{Q})$. The vertical arrows are the base change homomorphisms. By $[\mathrm{P} 2]$ Lemma $7.10 t$ is injective (the argument immediately extends to the case that $G$ is not cyclic). The right vertical arrow is also injective. We conclude that the left vertical arrow is injective; this implies the desired result: the $(d+1-i)$-extensions $E\left(\mathcal{L}^{(i)}, \xi^{(i)}\right)$ of $G^{D}$ by $\mathbf{G}_{m}$ (over $S=\operatorname{Spec}(\mathbf{Z})$ ) are trivial when $d+1-i \geq 3$. In addition, by [P2] Remark 7.8 (a) the $(d+1-i)$-extensions $E\left(\mathcal{L}^{(i)}, \xi^{(i)}\right)$ are trivial when $d+1-i<3$. This, together with $\operatorname{Pic}(S)=(0)$, implies that all the terms in the polynomial expansion of $\mathcal{L}^{\otimes(d+1) ! !}$ are trivial. Hence, by $(5.1)$, we have $2((d+1) ! !) \cdot \bar{\chi}^{P}\left(\mathcal{O}_{X}\right)=0$ in $\mathrm{Cl}(\mathbf{Z}[G])$. This concludes the proof of Theorem 1.5.

5.e. In this last paragraph we discuss the identity (1.1) of the Introduction. Let us first remark that a similar identity plays a crucial role in the proof of Theorem 3.3 ([P2] Theorem 8.4). Here we will explain the case $G=\mathbf{Z} / p \mathbf{Z}$ in some more detail (see also loc. cit. §8.d). Recall that we assume $p>d+1, p$ odd.

We set $\mathcal{L}=\operatorname{det} \mathbf{R} h_{G^{D}}\left(\pi_{*}\left(\mathcal{O}_{X}\right)\right)^{\otimes 2}$; by Theorem 4.7 this invertible sheaf over $G^{D}=\mu_{p}$ supports a canonical $d+2$-cubic structure $\xi$. The "polynomial expansion" of [P2], Cor. 5.6 now gives

$$
\mathcal{L}^{\otimes(d+1) ! !} \simeq \bigotimes_{i=0}^{d}\left(\Delta_{d+1-i}^{*}(E(d+1-i))^{\otimes(d-i) ! !}\right.
$$

where $E(j)$ is an invertible sheaf with a $j$-extension structure over $\mu_{p}^{j}$ and $\Delta_{j}: \mu_{p} \rightarrow \mu_{p}^{j}$ is the diagonal morphism. Since the invertible sheaves $\mathcal{L}$ and $E(j)$ are $p$-power torsion with $p>d+1$ and $p$ odd, we can write

$$
\operatorname{det} \mathbf{R} h_{G^{D} *}\left(\pi_{*}\left(\mathcal{O}_{X}\right)\right) \simeq \bigotimes_{j=1}^{d+1} \Delta_{j}^{*}\left(E^{\prime}(j)\right)
$$

where $E^{\prime}(j)$ is an invertible sheaf on $\mu_{p}^{j}$ with a $j$-extension structure. Now let $t_{j}(X / Y)$ be the image of the class of $E^{\prime}(j)$ under the isomorphism

$$
\psi_{j}: j-\operatorname{Ext}^{1}\left(\mu_{p}, \mathbf{G}_{m}\right) \rightarrow \operatorname{Hom}\left((C / p)^{(1-j)}, \mathbf{Z} / p \mathbf{Z}\right)
$$

of [P2] Proposition 7.5. We can now see that (5.9) and [P2] Cor. 8.2 implies that we have

$$
\bar{\chi}^{P}\left(\mathcal{O}_{X}\right)=\sum_{j=1}^{d+1} R^{(j)}\left(t_{j}(X / Y)\right)
$$

under Rim's isomorphism $\mathrm{Cl}(\mathbf{Z}[G]) \simeq \mathrm{Cl}\left(\mathbf{Q}\left(\zeta_{p}\right)\right)$.

Remark 5.7. a) The functoriality of the construction implies that the unramified $\mathbf{Z} / p \mathbf{Z}$ extensions of $\mathbf{Q}\left(\zeta_{p}\right)$ which are given by the $t_{i}(X / Y)$ 's only depend on the $\mathbf{Z} / p \mathbf{Z}$-cover of the generic fibers $X_{\mathbf{Q}} \rightarrow Y_{\mathbf{Q}}$.

b) Assume that the generic fibers $X_{\mathbf{Q}}, Y_{\mathbf{Q}}$ are smooth and geometrically irreducible. Then we can show that the $\mathbf{Z} / p \mathbf{Z}$-extension of $\mathbf{Q}\left(\zeta_{p}\right)$ that corresponds to the "top" class $t_{d+1}(X / Y)$, $d+1=\operatorname{dim}(X)$, admits the following explicit description: 
Set $V=X_{\mathbf{Q}\left(\zeta_{p}\right)}, W=Y_{\mathbf{Q}\left(\zeta_{p}\right)}$ for the base changes. Denote by $[D]$ the $p$-torsion class in $\operatorname{Pic}(W)$ that corresponds to the cover $V / W$. (This the image of the class of $V / W$ under the map $\mathrm{H}^{1}(W, \mathbf{Z} / p \mathbf{Z})=\mathrm{H}^{1}\left(W, \mu_{p}\right) \rightarrow{ }_{p} \operatorname{Pic}(W)$.) Consider the intersection $[D]^{d}=[D] \cap \cdots \cap[D]$ in the Chow group of 0 -cycles of $W$ and assume that $[D]^{d}$ is represented by a 0 -cycle $\sum_{i} n_{i} Q_{i}$. For each $i$ consider the unramified $\mathbf{Z} / p \mathbf{Z}$-extension $L_{i}$ of the residue field $K_{i}:=k\left(Q_{i}\right)$ which is obtained by specializing the cover $V / W$ at the point $Q_{i}$. This is a Kummer extension and we can write $L_{i}=K_{i}\left(a_{i}^{1 / p}\right)$, with $a_{i} \in K^{*}$. Then one can see that $t_{d+1}(X / Y)$ is the (Kummer) $\mathbf{Z} / p \mathbf{Z}$-extension of $\mathbf{Q}\left(\zeta_{p}\right)$ which is generated by the $p$-th root of

$$
\prod_{i}\left(\operatorname{Norm}_{K_{i} / \mathbf{Q}\left(\zeta_{p}\right)}\left(a_{i}\right)\right)^{-\frac{n_{i}}{(d+1) !}} \in \mathbf{Q}\left(\zeta_{p}\right)^{*} /\left(\mathbf{Q}\left(\zeta_{p}\right)^{*}\right)^{p} .
$$

(In particular, this is independent of the choice of the 0-cycle.) The details will appear elsewhere.

c) One should be able to view the $t_{i}(X / Y)$ 's as characteristic classes in a suitable algebraic cobordism theory of varieties with free $\mathbf{Z} / p \mathbf{Z}$-actions. To our knowledge such a theory has not been developed as of yet.

\section{APPENDIX}

In this appendix, we show how an argument due to Godeaux and Serre ([Se] §20) combined with an "arithmetic" version of Bertini's theorem (based on the theorem of Rumely on the existence of integral points [Ru], [MB2]) allows us to construct "geometric" $G$-torsors $\pi$ : $X \rightarrow Y$ with $Y$ regular and $Y \rightarrow \operatorname{Spec}(\mathbf{Z})$ projective and flat of relative dimension $d$ for any finite group $G$ and any integer $d \geq 1$. In fact, for the $G$-torsors that we construct $Y \rightarrow \operatorname{Spec}(\mathbf{Z})$ factors through a smooth morphism $Y \rightarrow \operatorname{Spec}\left(\mathcal{O}_{K}\right)$ where $\mathcal{O}_{K}$ is the ring of integers of a number field $K$.

To explain this set $L=\mathbf{Z}[G]$ and for an integer $r \geq 1$ we denote by $S(r)=\oplus_{m \geq 0} S(r)_{m}=$ $\operatorname{Sym}_{\mathbf{Z}}\left(L^{\oplus r}\right)$ the corresponding (graded) symmetric algebra with $G$-action. We set $X(r)=$ $\operatorname{Proj}(S(r))$; this is then a projective space of (relative) dimension $s=r \cdot(\# G)-1$ over $\operatorname{Spec}(\mathbf{Z})$ that supports a linear action of $G$. The quotient $Y(r)=X(r) / G$ is also a projective scheme: We can see that, for sufficiently large integers $k$,

$$
Y(r) \simeq \operatorname{Proj}\left(\bigoplus_{m \geq 0}\left(S(r)_{m k}\right)^{G}\right),
$$

and the graded algebra $\bigoplus_{m \geq 0}\left(S(r)_{m k}\right)^{G}$ is generated by the free $\mathbf{Z}$-module $M:=S(r)_{k}^{G}$. Set $\mathbf{P}(M)=\operatorname{Proj}(\operatorname{Sym}(M))$; then $Y(r)$ is a closed subscheme of $\mathbf{P}(M)$. (See [Se] $\S 20$ for the details of the argument in the corresponding situation over an algebraically closed field; the same argument readily applies to our case.) Denote by $\pi(r): X(r) \rightarrow Y(r)$ the quotient morphism. Let $B(r)$ be the closed subscheme of $X(r)$ consisting of points with non-trivial inertia subgroups and set $b(r)$ for the (reduced) image $\pi(r)(B(r))$. The group $G$ acts freely on the open subscheme $U(r):=X(r)-B(r)$, the morphism $\pi(r): U(r) \rightarrow V(r):=Y(r)-b(r)$ is a $G$-torsor and $V(r) \rightarrow \operatorname{Spec}(\mathbf{Z})$ is smooth of relative dimension $s$. One can now observe ([Se]) that for all sufficiently large $r$, each fiber of $b(r) \rightarrow \operatorname{Spec}(\mathbf{Z})$ has codimension $>d$ in the corresponding fiber of $Y(r) \rightarrow \operatorname{Spec}(\mathbf{Z})$. 
Now let us consider the dual projective space $\mathbf{P}\left(M^{\vee}\right)$ parametrizing hyperplane sections of $\mathbf{P}(M)$. Denote by $\mathbf{H} \subset \mathbf{P}(M) \times \mathbf{P}\left(M^{\vee}\right)$ the universal hyperplane section. Let us set $Q=\left(\mathbf{P}\left(M^{\vee}\right)\right)^{s-d}$. For $\phi: T \rightarrow Q, i=1, \ldots, s-d$, let $\mathbf{H}_{\phi}^{i}$ be the hyperplane in $\mathbf{P}(M) \times T$ that corresponds to $\operatorname{pr}_{i} \cdot \phi: T \rightarrow \mathbf{P}\left(M^{\vee}\right)$ (this is the Cartesian product

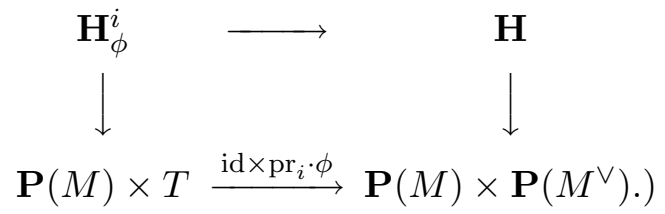

Set $Y(r)_{\phi: T \rightarrow Q}$ for the scheme theoretic intersection

$$
(Y(r) \times T) \cap\left(\mathbf{H}_{\phi}^{1} \cap \cdots \cap \mathbf{H}_{\phi}^{s-d}\right)
$$

in $\mathbf{P}(M) \times T$; this is a scheme over $T$. Let $V_{1}$ be the subset of $x \in Q$ for which

$$
Y(r)_{\operatorname{Spec}(k(x)) \rightarrow Q} \subset Y(r) \times \operatorname{Spec}(k(x))
$$

does not intersects $b(r) \times \operatorname{Spec}(k(x))$. Let $V_{2}$ be the subset of $x \in Q$ for which the projection $Y(r)_{\text {id: } Q \rightarrow Q} \rightarrow Q$ is flat at all points that lie over $x$. Finally, let $V_{3}$ be the subset of $x \in Q$ for which $Y(r)_{\operatorname{Spec}(k(x)) \rightarrow Q}$ is smooth over $\operatorname{Spec}(k(x))$ of dimension $d$. Set $V=V_{1} \cup V_{2} \cup V_{3}$; this is a constructible subset of $Q$. The proof of the usual Bertini theorem (over fields) applies to show that $V$ contains the generic point of each fiber $Q \rightarrow \operatorname{Spec}(\mathbf{Z})$. Therefore, the complement $Q-V$ is contained in a closed subscheme $Z$ which is such that $Q-Z \rightarrow \operatorname{Spec}(\mathbf{Z})$ is surjective. By $[\mathrm{Ru}]$ or $[\mathrm{MB}]$ there is a number field $K$ with integer ring $\mathcal{O}_{K}$ and an integral point $\phi: \operatorname{Spec}\left(\mathcal{O}_{K}\right) \rightarrow Q-Z \subset Q$. Since $Q-Z \subset V$, the pull-back of $X(r) \rightarrow Y(r)$ via $Y(r)_{\phi} \rightarrow Y(r) \times \operatorname{Spec}\left(\mathcal{O}_{K}\right)$ now gives a $G$-torsor $X \rightarrow Y:=Y(r)_{\phi}$ with $Y \rightarrow \operatorname{Spec}\left(\mathcal{O}_{K}\right)$ projective and smooth of relative dimension $d$. The argument in [Se] now shows that the generic fiber $X_{K}$ is geometrically connected.

\section{REFERENCES}

[BM] H. Bass, M. P. Murthy: Grothendieck groups and Picard groups of abelian group rings. Annals of Math. 86 (1967), 16-73.

[Bo] A. Borel: Stable real cohomology of arithmetic groups. Ann. Sci. École Norm. Sup. (4) 7 (1974), 235-272.

[Br] L. Breen: Fonctions thêta et théorème du cube. Lecture Notes in Mathematics, Vol. 980. SpringerVerlag, Berlin-New York, 1983.

[BCEM] J. Buhler, R. Crandall, R. Ernvall and T. Metsänkylä: Irregular primes and cyclotomic invariants to 12 million. Computational algebra and number theory (Milwaukee, WI, 1996). J. Symbolic Comput. 31 (2001), no. 1-2, 89-96.

[C] T. Chinburg: Galois structure of de Rham cohomology of tame covers of schemes, Annals of Math., 139 (1994), 443-490. Corrigendum, Annals of Math., 140 (1994), 251.

[CPT] T. Chinburg, G. Pappas, M. J. Taylor: Cubic structures, equivariant Euler characteristics and modular forms. Preprint. ArXiv math.NT/0309327

[DG] M. Demazure, P. Gabriel: Groupes algébriques. Masson et Cie, Paris; Amsterdam, North-Holland, 1970.

[De] P. Deligne: Le déterminant de la cohomologie, in Currents Trends in Arithmetical Algebraic Geometry. Contemporary Mathematics, Vol. 67, A.M.S. (1987). 
[Du] F. Ducrot: Cube structures and intersection bundles. J. Pure Appl. Algebra 195 (2005), no. 1, 33-73.

[F] A. Fröhlich: Galois Module Structure of Algebraic Integers. Ergebnisse der Mathematik und ihrer Grenzgebiete. (3) 1. Springer-Verlag, Berlin, 1983.

[KM] F. Knudsen, D. Mumford: The Projectivity of the Moduli space of Stable Curves (I): Preliminary on "det" and "Div". Math. Scand., 39 (1975), 19-55.

$[\mathrm{Ku}] \quad$ M. Kurihara: Some remarks on conjectures about cyclotomic fields and $K$-groups of $\mathbf{Z}$. Compositio Math. 81 (1992), no. 2, 223-236.

[La] S. Lang: Unramified class field theory over function fields in several variables. Ann. of Math. (2) 64 (1956), 285-325.

[MB] L. Moret-Bailly: Groupes de Picard et problémes de Skolem. I, II. Ann. Sci. École Norm. Sup. (4) 22 (1989), no. 2, 161-179, 181-194.

$[\mathrm{Mu}]$ D. Mumford. Abelian varieties. Tata Institute of Fundamental Research Studies in Mathematics, No. 5. Oxford University Press, London 1970 viii+242 pp.

[Na] S. Nakajima: On Galois module structure of the cohomology groups of an algebraic variety. Invent. Math. 75 (1984), no. 1, 1-8.

[P1] G. Pappas: Galois modules and the theorem of the cube. Invent. Math. 133 (1998), no. 1, 193-225.

[P2] G. Pappas: Cubic structures and ideal class groups. To appear in Ann. Sci. École Norm. Sup. Available at: http://www.math.msu.edu/ pappas/hCubicFin.pdf

[Ra] M. Raynaud: Spécialisation du foncteur de Picard. Inst. Hautes Études Sci. Publ. Math. No. 38, (1970), 27-76.

[Ri] D. S. Rim: Modules over finite groups. Annals of Math., 69 (1959), 700-712.

[Ro] J. Rognes: $K_{4}(\mathbf{Z})$ is the trivial group. Topology 39 (2000), no. 2, 267-281.

$[\mathrm{Ru}] \quad$ R. Rumely: Arithmetic over the ring of all algebraic integers. J. Reine Angew. Math. 368 (1986), 127-133.

[Se] J.-P. Serre: Sur la topologie des variétés algébriques en caractéristique $p .1958$ Symposium internacional de topologia algebraica (International symposium on algebraic topology) pp. 24-53. Universidad Nacional Autónoma de México and UNESCO, Mexico City. (Also in "J.-P. Serre, Collected Works", Springer-Verlag.)

[So] C. Soulé: Perfect forms and the Vandiver conjecture. J. Reine Angew. Math. 517 (1999), 209-221.

[SGA4] Théorie des topos et cohomologie étale des schémas. Dirigé par M. Artin, A. Grothendieck, et J. L. Verdier. Avec la collaboration de N. Bourbaki, P. Deligne et B. Saint-Donat. Lecture Notes in Mathematics, Vol. 269, 270, 305. Springer-Verlag, Berlin-New York, 1972-73.

[SGA6] Théorie des intersections et théorème de Riemann-Roch. Dirigé par P. Berthelot, A. Grothendieck et L. Illusie. Avec la collaboration de D. Ferrand, J. P. Jouanolou, O. Jussila, S. Kleiman, M. Raynaud et J. P. Serre. Lecture Notes in Mathematics, Vol. 225. Springer-Verlag, Berlin-New York, 1971.

[SGA7I] Groupes de monodromie en géométrie algébrique. I. Dirigé par A. Grothendieck. Avec la collaboration de M. Raynaud et D. S. Rim. Lecture Notes in Mathematics, Vol. 288. Springer-Verlag, Berlin-New York, 1972.

[Ta] M. J. Taylor: On Fröhlich's conjecture for rings of integers of tame extensions. Invent. Math. 63 (1981), no. 1, 41-79.

[W] L. Washington: Introduction to cyclotomic fields. Graduate Texts in Mathematics, 83. SpringerVerlag, New York, 1982. xi+389 pp.

School of Math., Institute for Advanced Study, Princeton, NJ 08540, U.S.A.,

(Permanent address: Dept. of Mathematics, Michigan State Univ., East Lansing, MI 48824, U.S.A.)

E-mail address: pappas@math.ias.edu, pappas@math.msu.edu 